MATHEMATICS OF COMPUTATION

Volume 72, Number 244, Pages 1769-1801

S 0025-5718(03)01538-2

Article electronically published on May 29, 2003

\title{
ANALYSIS OF VARIABLE-STEPSIZE LINEAR MULTISTEP METHODS WITH SPECIAL EMPHASIS ON SYMMETRIC ONES
}

\author{
B. CANO AND A. DURÁN
}

\begin{abstract}
In this paper we deal with several issues concerning variablestepsize linear multistep methods. First, we prove their stability when their fixed-stepsize counterparts are stable and under mild conditions on the stepsizes and the variable coefficients. Then we prove asymptotic expansions on the considered tolerance for the global error committed. Using them, we study the growth of error with time when integrating periodic orbits. We consider strongly and weakly stable linear multistep methods for the integration of first-order differential systems as well as those designed to integrate special second-order ones. We place special emphasis on the latter which are also symmetric because of their suitability when integrating moderately eccentric orbits of reversible systems. For these types of methods, we give a characterization for symmetry of the coefficients, which allows their construction, and provide some numerical results for them.
\end{abstract}

\section{INTRODUCTION}

In the recent literature, there is a lot of interest in achieving good variablestepsize integrators for Hamiltonian and reversible systems. For variable-stepsize one-step methods, asymptotic expansions of the global error have been deduced in [5] which allow a study on the growth of error with time when integrating periodic orbits. In the particular case when the system is reversible, symmetric variablestepsize one-step methods have proven to be an excellent tool [1], [5], 13, [19]. However, the fact that the stepsize selection must also be symmetric makes these methods implicit, requiring special techniques to reduce the additional work. The explicit techniques of [15] are based on a suitable reparameterization of time which allows fixed stepsizes to be taken; and for the case when the system is also Hamiltonian, a symmetric as well as symplectic method with fixed stepsizes applied over a modified Hamiltonian problem is suggested in [12, 17.

On the other hand, for fixed-stepsize linear multistep methods (FSLMM), an analysis has been given in 7] concerning the growth of error with time when integrating periodic orbits. For reversible systems, symmetric linear multistep methods for first-order differential systems were not recommended in general because they lead to exponential error growth. However, if the methods are especially designed

Received by the editor December 26, 2000 and, in revised form, April 30, 2002.

2000 Mathematics Subject Classification. Primary 65L06, 70F05, 70H33.

Key words and phrases. Linear multistep methods, variable stepsizes, stability, periodic orbits, error growth, reversible systems, symmetric integrators, asymptotic expansion of the error.

This work was supported by DGICYT PB95-705 and JCL VA36/98. 
for second-order differential systems in which the first derivative does not turn up (FSLMM2s), symmetric methods with no parasitic root in their first characteristic polynomial were very much recommended because they lead to advantageous error growth. These integrators, when explicit, make just one function evaluation per step.

The main aim of this paper is to analyze how variable-stepsize linear multistep methods (VSLMM) behave with respect to error growth. If the results are advantageous, the methods obtained should be competitive with the one-step techniques developed in [2] 12, 15, 17] when integrating some eccentric orbits because again explicit VSLMMs just make one function evaluation per step.

We emphasize that the analysis given in this paper is complete in several senses. In the first place, stability for VSLMMs has only been proved in the literature for some particular cases [3, 8, 10, all of them based on strongly stable integrators. We prove stability here for weakly as well as strongly stable methods assuming only some mild assumptions on the stepsizes and the variable coefficients. Secondly, asymptotic expansions of the global error on the stepsize or the tolerance have been proved in the literature for variable-stepsize one-step methods [5] and FSLMMs 6, 11, 14, 18, but not for VSLMMs, which we do here. Both facts, the stability and the new asymptotic expansions, are stated in section 2 and then proved in the appendix for the sake of clarity. We distinguish between VSLMMs to integrate first-order systems and some others designed to integrate special second-order ones (VSLMM2s). By using these asymptotic expansions, we analyze in section 3 the error growth with time when integrating periodic orbits of general and reversible systems with VSLMMs and VSLMM2s, completing in that way the study already initiated for other integrators and showing the good properties offered by some symmetric VSLMM2s to integrate reversible systems. Finally, to take advantage of this fact, we provide in section 4 the necessary and sufficient conditions for the coefficients of the method to make the VSLMM2 symmetric. This allows the construction of such methods in section 5 , which lets us corroborate experimentally the results proved in previous sections. The most practical conclusion drawn from the paper is stated in section 6.

We consider an initial value problem in $\mathbb{R}^{D}$ :

$$
\begin{aligned}
\dot{y}(t) & =f(y(t)), \\
y\left(t_{0}\right) & =\tilde{y}_{0},
\end{aligned}
$$

for a function $f$ which is $C^{\infty}$ in the bounded domain in which we are interested.

Given a tolerance $\epsilon$ to control the accuracy of the integration, the stepsize $h_{n}$ is usually $\epsilon s\left(y_{n}, \epsilon\right)$ for a function $s$ satisfying

- $s_{\min } \leq s(y, \epsilon) \leq s_{\max }$, with $s_{\min }, s_{\max }>0$,

- $s$ is $C^{\infty}$ in both arguments and all the derivatives of $s$ are bounded, for $\epsilon$ small enough and $y$ in the bounded domain. From this,

$$
h_{n}=\epsilon s\left(y\left(t_{n}\right), \epsilon\right)+O\left(\epsilon^{r}\right),
$$

where $r$ is the order of the method and $y\left(t_{n}\right)$ is the value approximated by $y_{n}$.

\section{Asymptotic EXPANSIONS OF GLOBAL ERROR}

2.1. First-order systems. Let us assume the initial value problem (1.1) is integrated with a VSLMM, i.e., a linear multistep method whose coefficients depend 
on the corresponding $k$ stepsizes satisfying the hypotheses in the introduction

$$
\sum_{l=0}^{k} \alpha_{l}\left(h_{n+k-1}, \ldots, h_{n}\right) y_{n+l}=h_{n+k-1} \sum_{l=0}^{k} \beta_{l}\left(h_{n+k-1}, \ldots, h_{n}\right) f\left(y_{n+l}\right) .
$$

Here $k$ is the number of steps of the method, $h_{m}$ denotes the stepsize considered to go from time $t_{m}$ to $t_{m+1}$ and $y_{m}$ denotes the numerical solution at time $t_{m}$. Notice that the coefficients $\left\{\alpha_{l}\right\},\left\{\beta_{l}\right\}$, which would be constants for a fixed-stepsize method, are now functions of the stepsizes. To globalize the already known fixedstepsize methods, we will assume $\alpha_{l}(h, \ldots, h)$ and $\beta_{l}(h, \ldots, h)$ are constants and do not depend on the stepsize $h$, and $\alpha_{k} \neq 0$. (Whenever we use the notation $\alpha_{l}, \beta_{l}$ without arguments, we will denote the constant fixed-stepsize ones.) On the other hand, we also assume

$$
\frac{\partial \alpha_{l}}{\partial h_{m}}(h, \ldots, h)=\frac{1}{h} \alpha_{l, m}^{D}, \quad \frac{\partial \beta_{l}}{\partial h_{m}}(h, \ldots, h)=\frac{1}{h} \beta_{l, m}^{D}, \quad m=0, \ldots, k-1,
$$

where $\partial / \partial h_{m}$ denotes the derivative with respect to the $(m+1)$-argument counting backwards, and where $\alpha_{l, m}^{D}$ and $\beta_{l, m}^{D}$ are certain constants which do not depend on $h$. This is not a serious restriction, as we will see, and together with the condition that the derivatives of order $p$ of $\alpha_{l}$ and $\beta_{l}$ are of order $O\left(1 / h^{p}\right)$ when evaluated at the same arguments allows the analysis given in this paper.

The method is determined by the combination of (2.1) and a starting procedure

$$
y_{\nu}=y\left(t_{\nu}\right)+\sum_{j=r}^{2 r-1} \epsilon^{j} s_{\nu}^{(j)}+O\left(\epsilon^{2 r}\right), \quad \nu=0,1, \ldots, k-1
$$

for given constants $s_{\nu}^{(j)}$.

Let us introduce now some notation before stating the main theorems of this section. We will call $\left\{x_{i}\right\}_{i=1}^{k}$ the roots of the first characteristic polynomial $\rho$, where

$$
\rho(x)=\sum_{l=0}^{k} \alpha_{l} x^{k}, \quad \sigma(x)=\sum_{l=0}^{k} \beta_{l} x^{k} .
$$

We will assume this fixed-stepsize method is irreducible and convergent and that it is normalized so that $\sigma(1)=1$. To be consistent, it is well known that $x_{1}=1$ must be one of these roots [11. On the other hand, for the method to be stable, it is necessary that all of these roots have modulus $\leq 1$ and those of modulus 1 be single [1]. We will let $\left\{x_{i}\right\}_{i=2}^{m}$ denote the $m-1$ roots of modulus 1 different from 1 . We assume the method $(\rho, \sigma)$ to be convergent of order $r$ and that its variable-stepsize counterpart is consistent of the same order, so that (2.1) is exact when $y_{n}$ is $y\left(t_{n}\right)$, with $y$ a polynomial of degree $\leq r$.

The following lemma will be used in the proof of the consecutive theorem and, at the same time, assures the stability of these methods. It suffices to apply it to the difference of two perturbations of (2.1) taking into account that $f$ is regular enough.

Lemma 2.1. Assume the sequence $\left\{z_{m}\right\}$ satisfies

$$
\begin{array}{r}
\alpha_{k}\left(h_{n+k-1}, \ldots, h_{n}\right) z_{n+k}+\cdots+\alpha_{0}\left(h_{n+k-1}, \ldots, h_{n}\right) z_{n} \\
=h_{n+k-1}\left(\beta_{k, n} z_{n+k}+\cdots+\beta_{0, n} z_{n}\right)+\lambda_{n}
\end{array}
$$


for given sequences $\left\{\beta_{l, n}\right\}_{l=0}^{k}$ which are uniformly bounded in $n,\left\{\lambda_{n}\right\}$ and $\left\{z_{\nu}\right\}_{\nu=0}^{k-1}$ also bounded in this way

$$
\begin{aligned}
& \left|z_{\nu}\right| \leq Z, \quad \nu=0,1, \ldots, k-1 \\
& \left|\lambda_{n}\right| \leq \Lambda, \quad n=0,1, \ldots, M
\end{aligned}
$$

and $h_{n}$ satisfying (1.2). Then, for $\epsilon$ small enough,

$$
\left|z_{n}\right| \leq C(M \Lambda+Z) e^{n \epsilon L}, \quad n=0,1, \ldots, M
$$

for given constants $C$ and $L$ which depend on the bounds for the $\left\{\beta_{l, n}\right\}$, on the function $s$ which determines the stepsizes and on a Lipschitz constant for $f$.

The following theorem determines the asymptotic expansion of the global error when integrating with a VSLMM.

Theorem 2.2. Under the hypotheses given above, the difference between the numerical procedure (2.1), 2.3) and the exact solution of (1.1) is

$$
y_{n}-y\left(t_{n}\right)=\sum_{j=r}^{2 r-1} \epsilon^{j} \sum_{i=1}^{m} x_{i}^{n} e_{j i}\left(t_{n}\right)+O\left(\epsilon^{2 r}\right), \quad \epsilon \rightarrow 0, n>0
$$

for some $C^{\infty}$-functions $\left\{e_{j i}\right\}_{j=r}^{2 r-1}$ satisfying

$$
\dot{e}_{j i}=\lambda_{i}\left(f^{\prime}(y(t)) e_{j i}(t)+h_{j i}(t)\right)+b_{j i}(t), \quad i=1, \ldots, m
$$

where $b_{r 1}(t)=-c_{r} y^{(r+1)}(t)$ for a certain constant $c_{r}$ and the rest of the functions $b_{j i}(t)$ depend on the exact solution, on the function $s$ which determines the stepsize, on the coefficients of the method, and for $i=2, \ldots, m$ also on $\left\{e_{l i}(t)\right\}_{l=r}^{j-1}$ and their derivatives. Moreover, $h_{j 1}=0$, and for $i=2, \ldots, m, h_{j i}(t)$ depends on the exact solution, on the function $s$ which determines the stepsize and on $\left\{e_{l i}(t)\right\}_{l=r}^{j}$ and their derivatives. On the other hand, the growing parameters $\lambda_{i}$ are calculated as

$$
\lambda_{i}=\frac{\sigma\left(x_{i}\right)}{x_{i} \rho^{\prime}\left(x_{i}\right)}, \quad i=1, \ldots, m \text {. }
$$

The implicit constant in the residual $O\left(\epsilon^{2 r}\right)$ can be chosen independently of $t_{n}$ for $t_{n}$ in each compact interval $\left[\delta, t_{\max }\right]$ where $t_{0}<\delta<t_{\max }$.

The proof of this theorem will be provided in the appendix.

Remark 2.3. For FSLMMs, we recall that $b_{j 1}(t)=-c_{j} y^{(j+1)}(t)(j=r, \ldots, 2 r-1)$ 6, 14, 18 for certain constants $c_{j}$. Therefore, in this case, the analysis of $e_{j 1}(t)$ is easier. Also in this case, $h_{j i}(t)=0(i=2, \ldots, m, j=r, \ldots, 2 r-1)$, simplifying the analysis for the corresponding $e_{j i}$ too.

Remark 2.4. In the proof of this theorem we will notice that $b_{j 1}$ is periodic whenever the solution to (1.1) is periodic. 
2.2. Second-order systems. Consider now a particular example of (1.1) associated to a second-order differential system of the type

$$
\begin{aligned}
\ddot{Y}(t) & =F(Y(t)), \\
Y\left(t_{0}\right) & =\tilde{y}_{0}, \\
\dot{Y}\left(t_{0}\right) & =\tilde{v}_{0},
\end{aligned}
$$

with $\tilde{y}_{0}, \tilde{v}_{0} \in \mathbb{R}^{d}(D=2 d)$. Fixed-stepsize linear multistep methods especially designed to integrate problems of this type (FSLMM2s) are well known [11. A natural generalization for variable stepsizes is

$$
\sum_{l=0}^{k} A_{l}\left(h_{n+k-1}, \ldots, h_{n}\right) Y_{n+l}=h_{n+k-1}^{2} \sum_{l=0}^{k} B_{l}\left(h_{n+k-1}, \ldots, h_{n}\right) F\left(Y_{n+l}\right) .
$$

We will again denote by $A_{l}$ and $B_{l}$ the corresponding coefficients associated to fixed stepsize and by $R$ and $S$ the first and second characteristic polynomials associated to (2.7) for fixed stepsize, with $A_{k} \neq 0$ and normalized so that $S(1)=1$. We will assume again that the coefficients $A_{l}()$ and $B_{l}()$ satisfy

$$
\frac{\partial A_{l}}{\partial h_{m}}(h, \ldots, h)=\frac{1}{h} A_{l, m}^{D}, \quad \frac{\partial B_{l}}{\partial h_{m}}(h, \ldots, h)=\frac{1}{h} B_{l, m}^{D},
$$

as well as the fact that the derivatives of order $p$ are $O\left(1 / h^{p}\right)$ when evaluated at the same arguments. Now, for the method to be consistent, it is necessary that $x_{1}=1$ be a double root of $R$, and for the method to be stable, that all the roots of $R$ have modulus $\leq 1$ and those of modulus 1 have multiplicity $\leq 2$. We will call $\left\{x_{i}\right\}_{i=2}^{m}$ the $m-1$ double roots of modulus 1 different from $1,\left\{x_{i}\right\}_{i=m+1}^{m+l}$ the $l$ single roots of modulus 1 different from 1 , and $R_{1}$ and $R_{2}$ the following polynomials

$$
R_{1}(x)=\prod_{i=1}^{m}\left(x-x_{i}\right), \quad R_{2}(x)=\frac{R(x)}{R_{1}(x)} .
$$

We will assume the method $(R, S)$ to be convergent of order $r$ and its variablestepsize counterpart also to be consistent of that order, so that (2.7) is exact when $Y_{n}$ is $Y\left(t_{n}\right)$, with $Y$ a polynomial of degree $\leq r+1$.

Again, the following lemma will be needed in the proof of the consecutive theorem on asymptotic expansions for VSLMM2s, as well as for assuring their stability.

Lemma 2.5. Assume the sequence $\left\{Z_{m}\right\}$ satisfies

$$
\begin{array}{r}
A_{k}\left(h_{n+k-1}, \ldots, h_{n}\right) Z_{n+k}+\cdots+A_{0}\left(h_{n+k-1}, \ldots, h_{n}\right) Z_{n} \\
=h_{n+k-1}^{2}\left[B_{k, n} Z_{n+k}+\cdots+B_{0, n} Z_{n}\right]+\lambda_{n},
\end{array}
$$

for given sequences $\left\{B_{l, n}\right\}_{l=0}^{k}$ which are uniformly bounded in $n,\left\{\lambda_{n}\right\}$ and $\left\{Z_{\nu}\right\}_{\nu=0}^{k-1}$ satisfying

$$
\begin{aligned}
Z_{\nu} & =O\left(\epsilon^{r}\right), \quad \nu=0,1, \ldots, k-1, \\
R_{2}(E) Z_{\nu} & =O\left(\epsilon^{r+1}\right), \quad \nu=0,1, \ldots, k-1, \\
\lambda_{n}=O\left(\epsilon^{r+2}\right), \quad \epsilon & \rightarrow 0, \quad \text { uniformly for } 0 \leq n \epsilon \leq t_{\max },
\end{aligned}
$$

for a given value $t_{\max }$ and $h_{n}$ satisfying $h_{n}=\epsilon s\left(Y\left(t_{n}\right), \epsilon\right)+O\left(\epsilon^{r}\right)$.

Then, $Z_{n}=O\left(\epsilon^{r}\right)$ uniformly for $0 \leq n \epsilon \leq t_{\max }$. 
Theorem 2.6. Under the hypotheses given above for a VSLMM, and taking starting values satisfying (2.3) as well as

$$
R_{2}(E)\left[Y_{l}-Y\left(t_{l}\right)\right]=O\left(\epsilon^{r+1}\right), \quad l=0,1, \ldots, m-1,
$$

the difference between the exact solution of (2.6) and the numerical procedure (2.7) satisfies

$$
Y_{n}=Y\left(t_{n}\right)+\sum_{j=r}^{2 r-1} \epsilon^{j} \sum_{i=1}^{m+l} x_{i}^{n} E_{j i}\left(t_{n}\right)+O\left(\epsilon^{2 r}\right), \quad \epsilon \rightarrow 0, n>0 .
$$

Here, for $i=1, \ldots, m,\left\{E_{j i}\right\}_{j=r}^{2 r-1}$ are $C^{\infty}$ - functions satisfying

$$
\ddot{E}_{j i}(t)=\mu_{i}\left(F^{\prime}(Y(t)) E_{j i}(t)+H_{j i}(t)\right)+B_{j i}(t), \quad i=1, \ldots, m
$$

where $F$ is the function in (2.6), $B_{r 1}(t)=-C_{r} Y^{(r+2)}(t)$ ( $C_{r}$ a constant) and the rest of the functions $B_{j i}$ depend on the exact solution, on the function $s$ which determines the stepsize, on the coefficients of the method and, for $i=2, \ldots, m$, also on $\left\{E_{l i}(t)\right\}_{l=r}^{j-1}$ and their derivatives. Moreover, $H_{j 1}(t)=0$ and, for $i=$ $2, \ldots, m, H_{j i}(t)$ depends on the exact solution, on the function $s$ which determines the stepsize, on the coefficients of the method, and on $\left\{E_{l i}(t)\right\}_{l=r}^{j}$ as well as their derivatives. The growing parameters $\mu_{i}$ are calculated as

$$
\mu_{i}=\frac{2 R\left(x_{i}\right)}{x_{i}^{2} S^{\prime \prime}\left(x_{i}\right)}, \quad i=1, \ldots, m
$$

For $i=m+1, \ldots, m+l$, the functions $E_{j i}$ satisfy

$$
\begin{aligned}
\alpha_{0, i}^{1}(t) E_{j i}(t)+\alpha_{1, i}^{1}(t) \dot{E}_{j i}(t)= & -\sum_{l=2}^{j+1-r} \sum_{m=0}^{l} \alpha_{m, i}^{l}(t) E_{j+1-l, i}^{(m)}(t) \\
& +\sum_{l=0}^{j-r-1} \sum_{m=0}^{l} \beta_{m, i}^{l}(t) \frac{d^{m}}{d t^{m}}\left(F^{\prime}(Y(t)) E_{j-l-1, i}(t)\right),
\end{aligned}
$$

where

$$
\begin{aligned}
& \alpha_{0, i}^{1}(t)=\frac{d}{d t} s(Y(t), 0)\left(\sum_{l=0}^{k} x_{i}^{l} \sum_{m=0}^{k-1} A_{l, m}^{D} m\right), \\
& \alpha_{1, i}^{1}(t)=s(Y(t), 0) R^{\prime}\left(x_{i}\right) x_{i},
\end{aligned}
$$

and the rest of the functions $\alpha_{m, i}^{l}$ and $\beta_{m, i}^{l}$ depend also on $s$, on the exact solution and on the coefficients of the method. The functions $E_{j i}$ are determined by equations (2.13) and (2.14) as well as the initial conditions given by the following linear systems

$$
-s_{\nu}^{(r)}+E_{r 1}\left(t_{0}\right)+\sum_{i=2}^{m} x_{i}^{\nu} E_{r i}\left(t_{0}\right)+\sum_{i=m+1}^{k-m} x_{i}^{\nu} E_{r i}\left(t_{0}\right)=0, \quad \nu=0,1, \ldots, k-1
$$




$$
\begin{aligned}
& \sum_{i=1}^{m} x_{i}^{\nu} E_{j i}\left(t_{0}\right)+\bar{t}_{\nu, 1,1} \sum_{i=1}^{m} x_{i}^{\nu} \dot{E}_{j-1, i}\left(t_{0}\right)+\sum_{i=m+1}^{k-m} x_{i}^{\nu} E_{j i}\left(t_{0}\right) \\
&= s_{\nu}^{(j)}-\sum_{l=2}^{j-r} \sum_{p=1}^{l} \frac{\bar{t}_{\nu, p, l}}{p !} E_{j-l, 1}^{(p)}\left(t_{0}\right)-\sum_{i=2}^{m} x_{i}^{\nu} \sum_{l=2}^{j-r} \sum_{p=1}^{l} \frac{\bar{t}_{\nu, p, l}}{p !} E_{j-l, i}^{(p)}\left(t_{0}\right) \\
&-\sum_{i=m+1}^{k-m} x_{i}^{\nu} \sum_{l=1}^{j-r} \sum_{p=1}^{l} \frac{\bar{t}_{\nu, p, l}}{p !} E_{j-l, i}^{(p)}\left(t_{0}\right), \\
& \sum_{j=0}^{k-m} \gamma_{j}\left(\sum_{i=m+1}^{k-m} x_{i}^{\nu+j} E_{2 r, i}\left(t_{0}\right)+\bar{t}_{\nu+j, 1,1} \dot{E}_{2 r-1,1}\left(t_{0}\right)\right. \\
&+\bar{t}_{\nu+j, 1,1} \sum_{i=2}^{m} x_{i}^{\nu+j} \dot{E}_{2 r-1, i}\left(t_{0}\right) \\
&+\sum_{l=2}^{r} \sum_{l=1}^{p} \frac{\bar{t}_{\nu+j, p, l}^{l}}{p !} E_{2 r-l, 1}^{(p)}\left(t_{0}\right) \\
&+\sum_{i=2}^{m} x_{i}^{\nu+j} \sum_{l=2}^{r} \sum_{p=1}^{l} \frac{\bar{t}_{\nu+j, p, l}}{p !} E_{2 r-l, i}^{(p)}\left(t_{0}\right) \\
&\left.+\sum_{i=m+1}^{k-m} x_{i}^{\nu+j}\left(\sum_{l=1}^{r} \sum_{p=1}^{l} \frac{\bar{t}_{\nu+j, p, l}}{p !} E_{2 r-l, i}^{(p)}\left(t_{0}\right)\right)-s_{\nu+j}^{(2 r)}\right)=0, \\
& \nu=0,1, \ldots, m-1,
\end{aligned}
$$

where $s_{\nu}^{(j)}$ come from the starting procedure (2.3), $\left\{\gamma_{j}\right\}$ are the coefficients of $R_{2}$ and $\bar{t}_{\nu, p, l}$ is the coefficient of $\epsilon^{l}$ in the expansion of $\left(t_{\nu}-t_{0}\right)^{p}$.

The implicit constant in the residual $O\left(\epsilon^{2 r}\right)$ can be chosen independently of $t_{n}$ for $t_{n}$ in each compact subinterval $\left[\delta, t_{\max }\right]$ where $t_{0}<\delta<t_{\max }$.

Remark 2.7. The proof of Theorem [2.6 will show that the functions $B_{j 1}, \alpha_{m, i}^{l}$ and $\beta_{m, i}^{l}$ are periodic whenever the solution to (2.6) is periodic.

Remark 2.8. We notice that, for FSLMM2s, $H_{j i}$ vanishes, $B_{j 1}=-C_{j} Y^{(j+2)}, \alpha_{0, i}^{1}$ in (2.15) vanishes and $\alpha_{m, i}^{l}, \beta_{m, i}^{l}$ are constants [6, 14, 18, simplifying the analysis.

Remark 2.9. From (2.14) and (2.15) we remark that

$$
k_{i} \frac{d}{d t} s(Y(t), 0) E_{r i}(t)+s(Y(t), 0) \dot{E}_{r i}(t)=0,
$$

for a certain constant $k_{i}$ which just depends on the coefficients of the method and does not vary with the stepsize taken. The solution for this is

$$
E_{r i}(t)=E_{r i}\left(t_{0}\right) e^{-k_{i}\left[\ln (s(Y(t), 0))-\ln \left(s\left(Y\left(t_{0}\right), 0\right)\right)\right]} .
$$

This will be used in section 3 .

The following theorem, also proved in section 6 , will be helpful in the construction of VSLMM2s. It simplifies the conditions under which order $r$ can be assured for these methods, in such a way that the previous theorems can be applied with that order. 
Theorem 2.10. Let us assume that a VSLMM2 has order of consistency $\geq r-1$ in the sense that the formula is exact for polynomials of degree $\leq r$ but has order $r$ with fixed stepsize. Then, in fact, the method has order of consistency $r$ with variable stepsizes and all the analysis given above would be true for that order.

Remark 2.11. If a symmetric VSLMM2 has order of consistency $\geq r-2$ and order $r$ with fixed stepsize ( $r$ even), then the method also has order of consistency $r$ with variable stepsizes.

Proof. It suffices to apply Theorem 2.10 with $r$ substituted by $r-1$ and to take into account that symmetric methods always have even order [11].

\section{ERror Growth IN THE INTEGRATION OF PERIODIC ORBITS}

By using the results in the previous section, we analyze here the error growth with time when integrating periodic orbits of general and reversible systems with VSLMMs and VSLMM2s.

3.1. First-order systems. In this subsection we will concentrate on initial value problems of the form (1.1) whose solution is $T$-periodic.

3.1.1. Strongly stable methods. In this paragraph, we consider the case in which the linear multistep method is strongly stable, i.e., all roots of $\rho$ have modulus $\leq 1$ and 1 is the only root of unit modulus. We see that, in that case, the asymptotic expansion given by Theorem 2.2 is

$$
y_{n}=y\left(t_{n}\right)+\sum_{j=r}^{2 r-1} \epsilon^{j} e_{j 1}\left(t_{n}\right)+O\left(\epsilon^{2 r}\right), \quad \epsilon \rightarrow 0, \quad n>0,
$$

with

$$
e_{j 1}^{I}(t)=\int_{t_{0}}^{t} M(t, s) b_{j 1}(s) d s, \quad e_{j 1}^{I I}(t)=M\left(t, t_{0}\right) e_{j 1}^{I I}\left(t_{0}\right) .
$$

Here $M(t, s)$ is the transition matrix associated with the homogeneous variational equation

$$
\dot{\delta}(t)=f^{\prime}(y(t)) \delta(t)
$$

To study how these coefficients grow with time we introduce the notation

$$
\begin{aligned}
& e_{j 1}[N]=e_{j 1}\left(t_{0}+N T\right), \\
& e_{j 1}^{I}[N]=e_{j 1}^{I}\left(t_{0}+N T\right), \\
& e_{j 1}^{I I}[N]=e_{j 1}^{I I}\left(t_{0}+N T\right),
\end{aligned}
$$

for the values of $e_{j 1}, e_{j 1}^{I}, e_{j 1}^{I I}$ after a whole number $N \geq 0$ of periods. An important observation is that the growth of $e_{j 1}(t)$ as a function of $t$ is essentially determined by the growth of $e_{j 1}[N]$ as a function of $N$. We see that, if $t \in\left[t_{0}+(N-1) T, t_{0}+N T\right]$, then

$$
e_{j 1}^{I I}(t)=M\left(t-(N-1) T, t_{0}\right) e_{j 1}^{I I}\left(t_{0}+(N-1) T\right),
$$

so that $e_{j 1}^{I I}(t)$ grows like $e_{j}^{I I}\left(t_{0}+(N-1) T\right)$ because $M\left(t-(N-1) T, t_{0}\right)$ is uniformly bounded in view of the bound $\left|t-(N-1) T-t_{0}\right| \leq T$. As for $e_{j 1}^{I}$, the following lemma also shows that the behaviour of $e_{j 1}^{I}(t)$ with time is very much related to the behaviour of $e_{j 1}^{I}[N-1]$ with $N$. 
Lemma 3.1. If $t \in\left[t_{0}+(N-1) T, t_{0}+N T\right]$, then, for $r \leq j \leq 2 r-1$,

$$
\begin{aligned}
e_{j 1}^{I}(t)= & M\left(t-(N-1) T, t_{0}\right) e_{j 1}^{I}\left(t_{0}+(N-1) T\right) \\
& +\int_{t_{0}}^{t-(N-1) T} M(t-(N-1) T, s) b_{j 1}(s) d s .
\end{aligned}
$$

Proof. The proof is similar to that of Lemma 1 in [7], where $b_{j 1}$ was substituted by its fixed-stepsize counterpart $-c_{j} y^{(j+1)}$, and follows from the periodicity of $b_{j 1}$ (Remark 2.4) and the properties of transition matrices.

Let us introduce now the notation $M_{t_{0}}$ for the monodromy matrix associated to the periodic problem (1.1). Then, from the previous lemma and (3.1), the following result follows:

Theorem 3.2. With the preceding notation,

$$
\begin{aligned}
e_{j 1}^{I}[N] & =M_{t_{0}} e_{j 1}^{I}[N-1]+e_{j 1}^{I}[1], \\
e_{j 1}^{I I}[N] & =M_{t_{0}} e_{j 1}^{I I}[N-1],
\end{aligned}
$$

and therefore,

$$
\begin{aligned}
& e_{j 1}^{I}[N]=\left(\sum_{l=0}^{N-1} M_{t_{0}}^{l}\right) e_{j 1}^{I}[1], \\
& e_{j 1}^{I I}[N]=M_{t_{0}}^{N-1} e_{j 1}^{I I}[1]=M_{t_{0}}^{N} e_{j 1}^{I I}[0] .
\end{aligned}
$$

The growth of the coefficients is therefore given by the growth of the corresponding powers of the monodromy matrix. To study that, we consider the following lemma whose proof is already in [5].

Lemma 3.3. Assume that $M$ is a $\nu \times \nu$ Jordan block with eigenvalue $\lambda$. Then, as $N \uparrow \infty$ :

(i) If $|\lambda| \geq 1, \lambda \neq 1$, then

$$
\left\|\sum_{i=0}^{N-1} M^{i}\right\|=O\left(N^{\nu-1}|\lambda|^{N}\right), \quad\left\|M^{N}\right\|=O\left(N^{\nu-1}|\lambda|^{N}\right) .
$$

(ii) If $|\lambda|<1$, then

$$
\left\|\sum_{i=0}^{N-1} M^{i}\right\|=O(1), \quad\left\|M^{N}\right\|=o(1) .
$$

(iii) If $\lambda=1, \nu>1$, then

$$
\left\|\sum_{i=0}^{N-1} M^{i}\right\|=O\left(N^{\nu}\right), \quad\left\|M^{N}\right\|=O\left(N^{\nu-1}\right) .
$$

(iv) If $\lambda=1, \nu=1$, then

$$
\sum_{i=0}^{N-1} M^{i}=N, \quad M^{N}=1
$$

From the previous results we conclude: 
Theorem 3.4. If a T-periodic problem of the type (1.1) is integrated with a strongly stable VSLMM satisfying the hypotheses of Theorem 2.2, then the following exclusive possibilites arise:

(G1) The solution $y(\cdot)$ has a Floquet multiplier of modulus $>1$, or in other words the monodromy matrix $M_{t_{0}}$ has spectral radius $R>1$. Then, for $r \leq j \leq 2 r-1, N \rightarrow \infty, e_{j 1}^{I}[N]$ and $e_{j 1}^{I I}[N]$ are $O\left(N^{\nu-1} R^{N}\right)$, where $\nu$ is the size of the largest Jordan block of $M_{t_{0}}$ corresponding to the eigenvalues of modulus $R$.

(G2) All Floquet multipliers have modulus $\leq 1$. Denote by $\nu$ the size of the largest Jordan block of $M_{t_{0}}$ corresponding to eigenvalues $\neq 1$ of modulus 1 and denote by $\nu_{1}$ the size of the largest Jordan block of $M_{t_{0}}$ with eigenvalue $1\left(\nu_{1} \geq 1\right)$. Then, for $r \leq j \leq 2 r-1$,

$$
\left\|e_{j 1}^{I}[N]\right\|=O\left(N^{\bar{\nu}}\right), \quad \bar{\nu}=\max \left(\nu-1, \nu_{1}\right)
$$

while

$$
\left\|e_{j 1}^{I I}[N]\right\|=O\left(N^{\bar{\nu}^{\prime}}\right), \quad \bar{\nu}^{\prime}=\max \left(\nu-1, \nu_{1}-1\right) .
$$

Therefore, the error growth is polynomial.

The following particular case of (G2) deserves special attention.

$\left(\mathrm{G}^{\prime}\right)$ The periodic solution is hyperbolic and attracting, i.e., 1 is a simple Floquet multiplier and the remaining $D-1$ multipliers have modulus $<1$. This corresponds to case (G2) above with $\nu=0, \nu_{1}=1$. Therefore, $\left\|e_{j 1}^{I}[N]\right\|=O(N)$ and $\left\|e_{j 1}^{I I}[N]\right\|=O(1)$. If we decompose $e_{j 1}^{I}[N]$ according to eigenvectors and generalized eigenvectors of $M_{t_{0}}$, then the components of $e_{j 1}^{I}[N]$ corresponding to multipliers $\neq 1$ remain bounded by (ii) in Lemma 3.3. Therefore, the only component which grows linearly is the one associated to the eigenvalue 1 , that is to say, to the eigenvector $f\left(y_{0}\right)$. This means that the error committed is basically a phase error.

The situation $\left(\mathrm{G} 2^{\prime}\right)$ is generic: if a differential system has a periodic orbit in $\left(\mathrm{G} 2^{\prime}\right)$, then all neighbouring differential systems have a periodic orbit in $\left(\mathrm{G} 2^{\prime}\right)$.

Remark 3.5. Notice that the results given by Theorem 3.4for variable stepsizes are the same that were already proved in [7] for fixed stepsizes.

Reversible systems. Let us assume the system to integrate is reversible, i.e., there exists a linear involution $\Lambda$ in $R^{D}$, such that

$$
f(\Lambda x) \equiv-\Lambda f(x)
$$

for the function $f$ in (1.1). Let us also assume that the solution to (1.1) is symmetric, i.e., the corresponding trajectory in phase space intersects the invariant subspace $X_{+}$of $\Lambda$. In such a case, the monodromy matrix of the orbit has special properties which allow a favourable growth of the coefficients $e_{j 1}$ (even $j$ ) of the global error for every FSLMM [7]. For variable stepsizes, however, it is only possible to assure that good behaviour for the first term $e_{r 1}(t)$ and just in the case $r$ is even. We believe that is meaningful enough and therefore we state the following result.

Lemma 3.6. Assume an even-order strongly stable VSLMM is employed to integrate a reversible initial value problem (1.1), (3.3), where $\tilde{y}_{0} \in X_{+}$and the solution is a symmetric periodic orbit. Then, the coefficients $e_{r 1}^{I}(t)$ of the asymptotic expansion of the error satisfy

$$
M_{t_{0}} \Lambda e_{r 1}^{I}[1]=-e_{r 1}^{I}[1]
$$


Proof. Theorem 2.2 says that $b_{r 1}(t)=-c_{r} y^{(r+1)}(t)$, and the result follows in the same way as in the proof for fixed stepsizes and even indexes [7].

From the previous results, the lemma above and the special structure of $M_{t_{0}}$ in the reversible case ( $\lambda$ an eigenvalue implies the same for $\frac{1}{\lambda}[5]$ ), the following theorem arises:

Theorem 3.7. Assume that the solution of the reversible initial value problem (1.1), (3.3) is a symmetric periodic orbit. When integrating with a strongly stable VSLMM, the following possibilities arise:

(R1) There is a Floquet multiplier of modulus $\neq 1$. Then, $e_{j 1}[N](j=r, \ldots$, $2 r-1)$ grows exponentially with $N$.

(R2) Every Floquet multiplier has modulus 1. Then, $e_{j 1}[N](j=r, \ldots, 2 r-1)$ grows polynomially with $N$.

Let us consider the following particular cases of (R2):

$\left(\mathrm{R} 2^{\prime}\right)$ Every Floquet multiplier $\leq 1$ has Jordan blocks of size $\leq 2$ and the multiplier 1 only posseses trivial Jordan blocks (of size 1 ). Then, $e_{j 1}[N]$ grows linearly with $N$.

$\left(\mathrm{R} 2^{\prime \prime}\right)$ Every Floquet multiplier has Jordan blocks of size $\leq 2$, and for the multiplier 1 there are no generalized eigenvectors in the invariant subspace of $-\Lambda$ (let us call that space $X_{-}$). Then,

- If the method has odd order, the terms $e_{j 1}[N](j=r, \ldots, 2 r-1)$ grow quadratically.

- If the method has even order, $e_{r 1}[N]$ grows linearly and the $O\left(\epsilon^{r+1}\right)$ term grows quadratically.

Remark 3.8. Therefore, in case $\left(\mathrm{R} 2^{\prime \prime}\right)$ and even order of the method, linear error growth will be observed for the first periods while for longer times the $O\left(\epsilon^{r+1}\right)$-term will become dominant and quadratic error growth will be finally observed.

Remark 3.9. The case ( $\left.\mathrm{R} 2^{\prime \prime}\right)$ includes many examples found in applications, including the periodic solutions of Kepler's problem. A discussion may be seen in [7] and it is possible to determine those directions in which the first error term grows, in the same way it was done for fixed stepsizes (see [6]).

3.1.2. Weakly stable methods. Let us consider now the case in which the first characteristic polynomial has roots of unit modulus different from 1 . For the term $e_{j 1}$, the same results as for the strongly stable methods apply. However, for fixed stepsizes it was already shown in [7] that, except for some very particular integrators such as those cited in 9] or the explicit midpoint and trapezoidal rule [6], the growth of error with time is typically exponential, even though the powers of $M_{t_{0}}$ only grow polynomially. This is due to the fact that the terms $\left\{e_{j i}\right\}$ in Theorem 2.2 corresponding to roots different from 1 satisfy equation (2.5), where the parameter $\lambda_{i}$ is different from 1 and -1 in general, and therefore the system (2.5) has no relation whatsoever with the variational equation of the orbit being integrated. For fixed stepsize, $h_{j i}(t) \equiv 0$ and the results are already very bad. For variable stepsizes, this term is nonzero, which just worsens the situation.

In particular, as is well known, stable symmetric methods are weakly stable, and therefore, they cannot be recommended in general, even for the integration of reversible systems, in contrast to their good apparent geometric properties [20]. 
3.2. Second-order systems. Let us assume now that we integrate a $T$-periodic problem of the type (2.6) with a VSLMM2. In such a case, and under the hypotheses of Theorem [2.6, the asymptotic expansion (2.12) applies, and therefore, we must study the growth with time of the coefficients $E_{j i}(t)$ associated to it.

Let us begin with the terms $E_{j i}(t)$ associated to the double roots $\left\{x_{i}\right\}_{i=1}^{m}$. We can rewrite the variational equations (2.13) in first-order form as

$$
\begin{aligned}
\frac{d}{d t}\left(\begin{array}{c}
\frac{1}{\sqrt{\mu_{i}}} \dot{E}_{j i}(t) \\
E_{j i}(t)
\end{array}\right)= & \sqrt{\mu_{i}}\left(\begin{array}{cc}
0 & F^{\prime}(Y(t)) \\
I & 0
\end{array}\right)\left(\begin{array}{c}
\frac{1}{\sqrt{\mu_{i}}} \dot{E}_{j i}(t) \\
E_{j i}(t)
\end{array}\right) \\
& +\left(\begin{array}{c}
\sqrt{\mu_{i}} H_{j i}(t)+B_{j i}(t) \\
0
\end{array}\right),
\end{aligned}
$$

when $i=1, \mu_{1}=1$, and $H_{j 1}(t)=0$. Then, we can consider the transition and monodromy matrices of this first-order system and the corresponding Floquet multipliers and arrive at the same conclusions as in Theorem 3.4 for the coefficients $E_{j 1}(t)$ because of the periodicity of $\left(B_{j 1}(t), 0\right)^{T}$. However, for $i=2, \ldots, m$, the transition matrices for the system above do not relate to the Floquet multipliers of the periodic solution being studied, and therefore the coefficients $E_{j i}(i=2, \ldots, m)$ grow exponentially in general. Therefore, VSLMM2s with double roots $x_{i}$ of unit modulus, $x_{i} \neq 1$, cannot be recommended, in the same way that happened for fixed stepsizes 7].

Let us study then the behaviour of the functions $E_{j i}(i=m+1, \ldots, m+l)$ associated with single roots of unit modulus. The conclusions are the same as for their fixed-stepsize counterparts [7], although the functions are different.

Theorem 3.10. Given the situation of Theorem 2.6, and assuming that the solution of (2.6) is $T$-periodic, the functions $E_{j i}(t), j=r, \ldots, 2 r-1, i=m+1, \ldots, m+$ $l$, grow like $O\left(t^{j-r}\right)$.

If, in addition, the starting procedure is of order $r+1$, then for $i=m+1, \ldots$, $m+l, E_{r i} \equiv 0$ and $E_{j i}$ grows like $O\left(t^{j-r-1}\right), j=r+1, \ldots, 2 r-1$.

If, furthermore, the starting procedure is of order $r+2$, then for $i=m+1, \ldots$, $m+l, E_{r i}(t) \equiv E_{r+1, i}(t) \equiv 0$ and $E_{j i}$ grows like $O\left(t^{j-r-2}\right), j=r+2, \ldots, 2 r-1$.

Proof. Notice that (2.19) implies that $E_{r i}(t)$ as well as its derivatives are bounded when the solution is periodic. Proceeding then inductively on $j>r$, from (2.14) and 2.15 it follows that

$$
k_{i} \frac{d}{d t} s(Y(t), 0) E_{j i}(t)+s(Y(t), 0) \dot{E}_{j i}(t)=F_{j i}(t),
$$

for a function $F_{j i}$ which grows with time like $O\left(t^{j-r-1}\right)$. The solution to (3.4) is then

$$
\begin{aligned}
E_{j i}(t)= & E_{j i}\left(t_{0}\right) e^{-k_{i}\left[\ln s(Y(t), 0)-\ln s\left(Y\left(t_{0}\right), 0\right)\right]} \\
& +\int_{t_{0}}^{t} e^{-k_{i}[\ln s(Y(t), 0)-\ln s(Y(u), 0)]} \frac{F_{j i}(u)}{s(Y(u), 0)} d u,
\end{aligned}
$$

and from here it is obvious that $E_{j i}(t)=O\left(t^{j-r}\right)$.

If the starting procedure is of order $r+1$, then from (2.16) $E_{r i}\left(t_{0}\right)=0$, which implies $E_{r i}(t)=0$ and therefore $F_{r+1, i}(t)=0$. From here, $E_{r+1, i}(t)=O(1)$, and inductively again $E_{j i}(t)=O\left(t^{j-r-1}\right), j=r+1, \ldots, 2 r-1$. 
If the starting procedure is of order $r+2$, from (2.17) $E_{r+1, i}\left(t_{0}\right)=0$, which implies also $E_{r+1, i}(t)=0$ and $F_{r+2, i}(t)=0$. Therefore, $E_{r+2, i}(t)=O(1)$, and inductively again $E_{j i}(t)=O\left(t^{j-r-2}\right), j=r+2, \ldots, 2 r-1$.

Remark 3.11. Notice that the earlier process cannot be generalized to a starting procedure of greater order because, in that case, the corresponding equation (2.17) would not be homogeneous any more because of the coefficients $E_{j-l, 1}^{(p)}\left(t_{0}\right)(l, p \geq 2)$.

3.2.1. Reversible systems. We remind the reader that, whenever the function $F$ in the second-order problem (2.6) satisfies

$$
\bar{\Lambda} \circ F \circ \bar{\Lambda}=F,
$$

for a linear involutive map $\bar{\Lambda}$ in $R^{d}$, then the first-order system associated to this problem is reversible with respect to the involution $\Lambda$ in $R^{D}$

$$
\Lambda\left(\begin{array}{c}
V \\
Y
\end{array}\right)=\left(\begin{array}{c}
-\bar{\Lambda} V \\
\bar{\Lambda} Y
\end{array}\right)
$$

As the variational equations for $E_{j 1}$ (2.13) can be written in first-order form, the results obtained in Theorem 3.7 about $E_{j 1}$ also apply now for the involution $\Lambda$ and the monodromy matrix associated to the underlying first-order system.

Symmetric methods. Let us see now the advantages of considering a suitable symmetric VSLMM2 in order to integrate a reversible problem.

When considering variable stepsizes, we say that the method is symmetric if, whenever the method takes $Y_{n}, \ldots, Y_{n+k-1}$ to $Y_{n+k}$ with stepsizes $h_{n}, \ldots, h_{n+k-1}$, integrating backwards it takes $Y_{n+k}, \ldots, Y_{n+1}$ to $Y_{n}$ with stepsizes $h_{n+k-1}, \ldots, h_{n}$ 11. As the choice of the stepsize is also inherent to the method, to assure that the stepsize taken to go from $y_{n}$ to $y_{n+1}$ is the same as the one to go from $y_{n+1}$ to $y_{n}$, we can choose $h_{n}=\epsilon s\left(Y_{n}, \epsilon\right)$ and then impose

$$
s\left(Y_{n}, \epsilon\right)=s\left(Y_{n+1},-\epsilon\right) .
$$

We see that, for instance, this condition is satisfied if

$$
h_{n}=\frac{\epsilon}{2}\left[\tau\left(Y_{n}\right)+\tau\left(Y_{n+1}\right)\right]
$$

for some function $\tau$ [16].

As was shown by Stoffer [20], when a symmetric $k$ th-step method is applied to the solution of a $\bar{\Lambda}$-reversible system (3.5), the following happens: If $Y_{n}, Y_{n+1}, \ldots, Y_{n+k}$ are $k+1$ consecutive values of a numerical trajectory, then the same occurs with $\bar{\Lambda} Y_{n+k}, \bar{\Lambda} Y_{n+k-1}, \ldots, \bar{\Lambda} Y_{n}$. Notice that, for variable stepsizes, the result still applies together with the condition that

$$
s\left(Y_{n}, \epsilon\right) \equiv s\left(\bar{\Lambda} Y_{n+1}, \epsilon\right), \quad n>0 .
$$

As was shown in [5], this condition is satisfied when the function $\tau$ in (3.6) is such that

$$
\tau(Y)=\tau(\bar{\Lambda} Y)
$$

It is well known that symmetric FSLMM2s satisfy the condition that all the roots of the first characteristic polynomial $R$ have modulus 1 [7]. If they have more double roots than $x_{1}=1$, they cannot be recommended for reversible systems, for reasons which have also already been gone through for variable stepsizes. However, at least for fixed stepsizes, the methods with no parasitic root lead to very advantageous properties with respect to error growth. We are showing now that "nearly" the 
same happens for variable stepsizes, although the way to prove it is much different from that of their fixed-stepsize counterparts. This is due to the fact that, at least for $j>r$, the terms $B_{j 1}$ in (2.13) are not simple scalar multiples of successive derivatives of $\mathrm{Y}(\mathrm{t})$ when variable stepsizes are considered. In any case, the following lemma allows us to prove the consequent theorem, which determines the growth of error with time of the first three terms of the asymptotic expansion.

Let us initially assume that the starting procedure is symmetric with respect to the invariant subspace by $\bar{\Lambda}$ (denoted by $\bar{X}_{+}$), i.e.,

$$
\bar{\Lambda} Y_{\left[\frac{k}{2}\right]+l}=Y_{\left[\frac{k}{2}\right]-l}, \quad l=0,1, \ldots, k-\left[\frac{k}{2}\right]-p .
$$

Here $p=0$ if $k$ is even and $p=1$ if $k$ is odd. Notice that this condition just involves $Y_{0}, \ldots, Y_{k-1}$ for odd $k$ while it involves $Y_{0}, \ldots, Y_{k}$ for even $k$. For the latter, we can choose symmetric $Y_{1}, \ldots, Y_{k-1}$ and then select $Y_{0}$ from the condition $\bar{\Lambda} Y_{0}=Y_{k}$ and the equations (2.7) which determine the method.

Let us also assume $Y_{1}, \ldots, Y_{k-1}$ are exact as well as $Y_{0}$ in the odd- $k$ case. (Notice that in the even- $k$ case, $Y_{0}$ may not be exact but it will differ from $Y\left(t_{0}\right)$ in just $O\left(\epsilon^{r+2}\right)$ because of its construction.)

Lemma 3.12. Let us assume we integrate a reversible initial value problem (2.6), (3.5) whose solution is periodic and symmetric with an rth-order symmetric VSLMM2, whose fixed-stepsize counterpart has no double roots except for $x_{1}=1$. If the starting procedure is as considered above, the term $E_{j 1}^{\left(\left[\frac{k}{2}\right]\right)}[N]$ associated to the root 1 which multiplies $\epsilon^{j}$ in the asymptotic expansion of the global error (2.12) in time $N T+t_{\left[\frac{k}{2}\right]}$ satisfies

$$
-E_{j 1}^{\left(\left[\frac{k}{2}\right]\right)}[N]=\pi_{2} M_{t_{\left[\frac{k}{2}\right]}}^{N} \Lambda\left(\begin{array}{c}
\dot{E}_{j 1}^{\left(\left[\frac{k}{2}\right]\right)}[N]+I_{1, j} \\
E_{j 1}^{\left(\left[\frac{k}{2}\right]\right)}[N]+I_{2, j}
\end{array}\right), \quad r \leq j \leq r+2,
$$

for some values $I_{1, j}, I_{2, j}$ independent of $N$ and $\pi_{2}$ the projection on $\mathbb{R}^{d}$ of the lower-half components of a vector in $\mathbb{R}^{2 d}$. Furthermore, $I_{1, r}=I_{2, r}=I_{2, r+1}=0$ and $I_{1, r+1} \in \bar{X}_{+}$.

Proof. Let us denote as $\Psi_{\epsilon}$ the reversible integrator which, for any integer $l \geq 0$, $t_{\hat{N}}=N T$ and stepsizes associated to a tolerance $\epsilon$, takes the values $Y_{l}, \ldots, Y_{l+k-1}$ to $Y_{l+\hat{N}}, Y_{l+\hat{N}+1}, \ldots, Y_{l+\hat{N}+k-1}$. Then,

$$
\Psi_{\epsilon}\left(\begin{array}{c}
Y_{0} \\
Y_{1} \\
\vdots \\
Y_{k-1}
\end{array}\right)=\left(\begin{array}{c}
Y_{\hat{N}} \\
Y_{\hat{N}+1} \\
\vdots \\
Y_{\hat{N}+k-1}
\end{array}\right) .
$$

Because of the symmetry and therefore reversibility of the method,

$$
\Psi_{\epsilon}\left(\begin{array}{c}
\bar{\Lambda} Y_{\hat{N}+k-1} \\
\bar{\Lambda} Y_{\hat{N}+k-2} \\
\vdots \\
\bar{\Lambda} Y_{\hat{N}}
\end{array}\right)=\left(\begin{array}{c}
\bar{\Lambda} Y_{k-1} \\
\bar{\Lambda} Y_{k-2} \\
\vdots \\
\bar{\Lambda} Y_{0}
\end{array}\right) .
$$

The key point of the proof is that the starting procedure in (3.9) satisfies the hypotheses of Theorem 2.6] and therefore we can consider the asymptotic expansion 
of the global error committed when integrating the same problem (2.6), (3.5). On the other hand, as $Y_{[k / 2]} \in \bar{X}_{+}$and is exact, (3.9) is saying that the error committed at time $N T+t_{[k / 2]}$ is zero with these starting values. For both things to be true, (3.8) must be satisfied.

Consider the asymptotic expansions for the global error at $Y_{\hat{N}+l}$ when starting from $\left(Y_{0}, \ldots, Y_{k-1}\right)^{T}$. Then, the starting procedure in (3.9) can be written as

$$
\left(\begin{array}{c}
\bar{\Lambda} Y_{k-1}+\sum_{j=r}^{2 r-1} \epsilon^{j} \bar{\Lambda} E_{j}^{(k-1)}[N] \\
\bar{\Lambda} Y_{k-2}+\sum_{j=r}^{2 r-1} \epsilon^{j} \bar{\Lambda} E_{j}^{(k-2)}[N] \\
\vdots \\
\left.\bar{\Lambda} Y\left(t_{0}\right)+\sum_{j=r}^{2 r-1} \epsilon^{j} \bar{\Lambda} E_{j}^{(0)}[N]\right)
\end{array}\right)=\left(\begin{array}{c}
Y_{2\left[\frac{k}{2}\right]-k+1}+\sum_{j=r}^{2 r-1} \epsilon^{j} \bar{\Lambda} E_{j}^{(k-1)}[N] \\
Y_{2\left[\frac{k}{2}\right]-k+2}+\sum_{j=r}^{2 r-1} \epsilon^{j} \bar{\Lambda} E_{j}^{(k-2)}[N] \\
\vdots \\
\bar{\Lambda} Y\left(t_{2\left[\frac{k}{2}\right]}\right)+\sum_{j=r}^{2 r-1} \epsilon^{j} \bar{\Lambda} E_{j}^{(0)}[N]
\end{array}\right)
$$

except for an $O\left(\epsilon^{2 r}\right)$-term. Here $E_{j}^{(l)}[N]$ denotes the whole term which multiplies $\epsilon^{j}$ in the asymptotic expansion (2.12) after time $t_{\hat{N}}=N T$ with starting values $Y_{l}, \ldots, Y_{l+k-1}$, and the fact that the $\left(Y_{0}, \ldots, Y_{k-p}\right)^{T}$ have been chosen to be symmetric implies the equality.

Let us denote as $\tilde{E}_{j}[N]$ the whole coefficient of $\epsilon^{j}$ in the asymptotic expansion of the global error (2.12) after time $t_{\hat{N}}=N T$ with the starting values (3.10). (Notice that Theorem 2.6 can be considered for this expression due to the fact that the starting procedure has order $r$ and (2.11) also applies because 1 is a root of $R_{2}$ and $E_{r}(t)$ equals the differentiable function $E_{r 1}(t)$ in this case.) As already mentioned, $\tilde{E}_{j}^{\left(\left[\frac{k}{2}\right]\right)}=0$ for every value of $j$. It is convenient for us to write this as

$$
-E_{j}^{\left(\left[\frac{k}{2}\right]\right)}[N]=\tilde{E}_{j}^{\left(\left[\frac{k}{2}\right]\right)}[N]-E_{j}^{\left(\left[\frac{k}{2}\right]\right)}[N] .
$$

For $j=r$, the initial conditions for $\tilde{E}_{r i}^{\left(\left[\frac{k}{2}\right]\right)}-E_{r i}^{\left(\left[\frac{k}{2}\right]\right)}(i=1, \ldots, k-1)$ are given by the linear systems in (2.16) with $t_{0}$ substituted by $t_{[k / 2]}$, and taking into account that

$$
\tilde{s}_{\nu}^{(r)}-s_{\nu}^{(r)}=\bar{\Lambda} E_{r}^{\left(\left[\frac{k}{2}\right]\right)}[N], \quad \nu=0,1, \ldots, k-1 .
$$

In such a way,

$$
\begin{aligned}
& \left(\tilde{E}_{r 1}^{\left(\left[\frac{k}{2}\right]\right)}-E_{r 1}^{\left(\left[\frac{k}{2}\right]\right)}\right)\left(t_{\left[\frac{k}{2}\right]}\right)=\bar{\Lambda} E_{r}^{\left(\left[\frac{k}{2}\right]\right)}[N], \\
& \left(\tilde{E}_{r i}^{\left(\left[\frac{k}{2}\right]\right)}-E_{r i}^{\left(\left[\frac{k}{2}\right]\right)}\right)\left(t_{\left[\frac{k}{2}\right]}\right)=0, \quad i=2, \ldots, k-1 .
\end{aligned}
$$

On the other hand, from system (2.17) with

$$
\tilde{s}_{\nu}^{(r+1)}-s_{\nu}^{(r+1)}=\bar{\Lambda} E_{r+1}^{\left(\left[\frac{k}{2}\right]\right)}[N]-\bar{t}_{\nu, 1,1} \bar{\Lambda} \dot{E}_{r}^{\left(\left[\frac{k}{2}\right]\right)}[N], \quad \nu=0,1, \ldots, k-1,
$$

it follows that

$$
\begin{aligned}
\left(\tilde{E}_{r+1,1}^{\left(\left[\frac{k}{2}\right]\right)}-E_{r+1,1}^{\left(\left[\frac{k}{2}\right]\right)}\right)\left(t_{\left[\frac{k}{2}\right]}\right) & =\bar{\Lambda} E_{r+1}^{\left(\left[\frac{k}{2}\right]\right)}[N], \\
\left(\dot{\tilde{E}}_{r 1}^{\left(\left[\frac{k}{2}\right]\right)}-\dot{E}_{r 1}^{\left(\left[\frac{k}{2}\right]\right)}\right)\left(t_{\left[\frac{k}{2}\right]}\right) & =-\bar{\Lambda} \dot{E}_{r}^{\left(\left[\frac{k}{2}\right]\right)}[N], \\
\left(\tilde{E}_{r+1, i}^{\left(\left[\frac{k}{2}\right]\right)}-E_{r+1, i}^{\left(\left[\frac{k}{2}\right]\right)}\right)\left(t_{\left[\frac{k}{2}\right]}\right) & =0, \quad i=2, \ldots, k-1 .
\end{aligned}
$$


Therefore, by considering the homogeneous variational differential system satisfied by $\tilde{E}_{r 1}-E_{r 1}$,

$$
\left(\begin{array}{c}
\dot{\tilde{E}}_{r r}^{\left(\left[\frac{k}{2}\right]\right)}[N]-\dot{E}_{r 1}^{\left(\left[\frac{k}{2}\right]\right)}[N] \\
\tilde{E}_{r 1}^{\left(\left[\frac{k}{2}\right]\right)}[N]-E_{r 1}^{\left(\left[\frac{k}{2}\right]\right)}[N]
\end{array}\right)=M_{t_{\left[\frac{k}{2}\right]}^{N} \Lambda}^{N} \Lambda\left(\begin{array}{c}
\dot{E}_{r 1}^{\left(\left[\frac{k}{2}\right]\right)}[N] \\
E_{r 1}^{\left(\left[\frac{k}{2}\right]\right)}[N]
\end{array}\right),
$$

and (3.8) follows for $j=r$ from (3.11) and the second projection of this.

For $j=r+1$, we must still calculate $\left(\dot{\tilde{E}}_{r+1,1}^{\left(\left[\frac{k}{2}\right]\right)}-\dot{E}_{r+1,1}^{\left(\left[\frac{k}{2}\right]\right)}\right)\left(t_{\left[\frac{k}{2}\right]}\right)$ from (2.17) with

$$
\begin{aligned}
\tilde{s}_{\nu}^{(r+2)}-s_{\nu}^{(r+2)}= & \bar{\Lambda} E_{r+2}^{\left(\left[\frac{k}{2}\right]\right)}[N]-\bar{t}_{\nu, 1,1} \bar{\Lambda} \dot{E}_{r+1}^{\left(\left[\frac{k}{2}\right]\right)}[N]-\bar{t}_{\nu, 1,2} \bar{\Lambda}^{\left(\left[\frac{k}{2}\right]\right)}[N] \\
& +\bar{t}_{\nu, 2,2} \bar{\Lambda} \ddot{E}_{r}^{\left(\left[\frac{k}{2}\right]\right)}[N]+d_{\nu} Y^{(r+2)}\left(t_{\left[\frac{k}{2}\right]}\right), \quad \nu=0,1, \ldots, k-1,
\end{aligned}
$$

$d_{\nu}$ coming from the difference between $Y\left(t_{\nu+\left[\frac{k}{2}\right]}\right)$ and $Y_{\nu+\left[\frac{k}{2}\right]}\left(\nu \geq\left[\frac{k}{2}\right]\right)$ and which is independent of $N$. Besides,

$$
\left(\ddot{\tilde{E}}_{r 1}^{\left(\left[\frac{k}{2}\right]\right)}-\ddot{E}_{r 1}^{\left(\left[\frac{k}{2}\right]\right)}\right)\left(t_{\left[\frac{k}{2}\right]}\right)=F^{\prime}\left(Y\left(t_{\left[\frac{k}{2}\right]}\right)\right) \bar{\Lambda} E_{r}^{\left(\left[\frac{k}{2}\right]\right)}[N]
$$

and

From this,

$$
\ddot{E}_{r}^{\left(\left[\frac{k}{2}\right]\right)}[N]=F^{\prime}\left(Y\left(t_{\left[\frac{k}{2}\right]}\right)\right) E_{r}^{\left(\left[\frac{k}{2}\right]\right)}[N]-C_{r} Y^{(r+2)}\left(t_{\left[\frac{k}{2}\right]}\right) .
$$

$$
\begin{aligned}
\left(\tilde{E}_{r+2,1}^{\left(\left[\frac{k}{2}\right]\right)}-E_{r+2,1}^{\left(\left[\frac{k}{2}\right]\right)}\right)\left(t_{\left[\frac{k}{2}\right]}\right) & =\bar{\Lambda} E_{r+2,1}^{\left(\left[\frac{k}{2}\right]\right)}[N]+L_{2, r+2}, \\
\left(\dot{\tilde{E}}_{r+1,1}^{\left(\left[\frac{k}{2}\right]\right)}-\dot{E}_{r+1,1}^{\left(\left[\frac{k}{2}\right]\right)}\right)\left(t_{\left[\frac{k}{2}\right]}\right) & =-\bar{\Lambda} \dot{E}_{r+1,1}^{\left(\left[\frac{k}{2}\right]\right)}[N]+L_{1, r+1}, \\
\left(\tilde{E}_{r+2, i}^{\left(\left[\frac{k}{2}\right]\right)}-E_{r+2, i}^{\left(\left[\frac{k}{2}\right]\right)}\right)\left(t_{\left[\frac{k}{2}\right]}\right) & =\bar{\Lambda} E_{r+2, i}^{\left(\left[\frac{k}{2}\right]\right)}+G_{i, r+1},
\end{aligned}
$$

where

$$
\begin{aligned}
L_{j, r+2} & =l_{j, r+2} Y^{(r+2)}\left(t_{\left[\frac{k}{2}\right]}\right), \quad j=1,2, \\
G_{i, r+1} & =g_{i, r+1} Y^{(r+2)}\left(t_{\left[\frac{k}{2}\right]}\right), \quad i=2, \ldots, k-1,
\end{aligned}
$$

for scalars $l_{2, r+2}, l_{1, r+1}, g_{i, r+1}$ such that

$$
l_{2, r+2}+\bar{t}_{\nu, 1,1} l_{1, r+1}+\sum_{i=1}^{k-1} x_{i}^{\nu} g_{i, r+1}=d_{\nu}-\frac{\bar{t}_{\nu, 2,2}}{2} c_{r}, \quad \nu=0,1, \ldots, k-1 .
$$

As a consequence,

$$
\left(\begin{array}{c}
\dot{\tilde{E}}_{r+1,1}^{\left(\left[\frac{k}{2}\right]\right)}[N]-\dot{E}_{r+1,1}^{\left(\left[\frac{k}{2}\right]\right)}[N] \\
\tilde{E}_{r+1,1}^{\left(\left[\frac{k}{2}\right]\right)}[N]-E_{r+1,1}^{\left(\left[\frac{k}{2}\right]\right)}[N]
\end{array}\right)=M_{t_{\left[\frac{k}{2}\right]}^{N} \Lambda}^{N} \Lambda\left(\begin{array}{c}
\dot{E}_{r+1,1}^{\left(\left[\frac{k}{2}\right]\right)}[N]-\bar{\Lambda} L_{1, r+1} \\
E_{r+1,1}^{\left(\left[\frac{k}{2}\right]\right)}[N]
\end{array}\right)
$$

so that (3.8) is proved with $I_{1, r+1}=-\bar{\Lambda} L_{1, r+1}=-l_{1, r+1} Y^{(r+2)}\left(t_{\left[\frac{k}{2}\right]}\right)$, which belongs to $\bar{X}_{+}$because $Y\left(t_{\left[\frac{k}{2}\right]}\right) \in \bar{X}_{+}$, the orbit is symmetric and the order $r$ of a symmetric method is even.

For the case $j=r+2$, in a similar manner

$$
\left(\dot{\tilde{E}}_{r+2,1}-\dot{E}_{r+2,1}\right)\left(t_{\left[\frac{k}{2}\right]}\right)=-\bar{\Lambda} \dot{E}_{r+2,1}^{\left(\left[\frac{k}{2}\right]\right)}[N]+L_{1, r+2},
$$

with $L_{1, r+2}$ independent of $N$. Therefore, equating the smooth parts of (3.11),

$$
-E_{r+2,1}^{\left(\left[\frac{k}{2}\right]\right)}[N]=\tilde{E}_{r+2,1}^{\left(\left[\frac{k}{2}\right]\right)}[N]-E_{r+2,1}^{\left(\left[\frac{k}{2}\right]\right)}[N]=\pi_{2} M_{t_{\left[\frac{k}{2}\right]}^{N}}^{N}-\Lambda\left(\begin{array}{c}
\dot{E}_{r+2,1}^{\left(\left[\frac{k}{2}\right]\right)}[N]-\bar{\Lambda} L_{1, r+2} \\
E_{r+2,1}^{\left(\left[\frac{k}{2}\right]\right)}[N]+\bar{\Lambda} L_{2, r+2}
\end{array}\right) .
$$


Theorem 3.13. Let us assume we integrate a reversible initial value problem (2.6), (3.5) whose solution is periodic and symmetric with an rth-order symmetric VSLMM2, whose fixed-stepsize first characteristic polynomial counterpart has no double roots except for $x_{1}=1$. Let us also assume that the starting procedure (2.3) is of order $r+2$ and that, for the monodromy matrix $M_{t_{0}}$ and the involution $\Lambda$, situation (R2") applies. Then, the terms in the asymptotic expansion of the global error associated to $\epsilon^{r}, \epsilon^{r+1}$ and $\epsilon^{r+2}$ grow linearly.

Proof. After Theorem 3.10 on the growth of the coefficients $E_{j i}$ associated to the single roots of $R$, and taking into account that the term $E_{j 1}^{I I}$ in the decomposition of $E_{j 1}$ grows linearly because of Theorem 3.4 , the point to prove is that the coefficients $E_{j 1}^{I}$ also grow linearly with time.

Let us assume in principle that $\tilde{y}_{0} \in \bar{X}_{+}$.

As the functions $E_{j 1}^{I}$ do not depend on the starting procedure, it suffices to prove it for a particular one. So we consider the values $\hat{Y}_{0}, \ldots, \hat{Y}_{k-1}$ symmetrically (3.7) and nearly "exact" as in Lemma 3.12 imposing $\tilde{y}_{0}=\hat{Y}_{\left[\frac{k}{2}\right]}$. Then, we take

$$
Y_{l}=\hat{Y}_{\left[\frac{k}{2}\right]+l}, \quad l=0, \ldots, k-1-\left[\frac{k}{2}\right],
$$

and for $l=k-\left[\frac{k}{2}\right], \ldots, k-1$, we consider the numerical values obtained when advancing the integration with our VSLMM2s from $\hat{Y}_{0}, \ldots, \hat{Y}_{k-1}$. In such a way, the starting procedure $\left(Y_{0}, \ldots, Y_{k-1}\right)$ has order $r+2$ (the same as the local truncation error of the method), and the smooth coefficients of the global error associated to it are those in (3.8). To simplify the notation at this moment, we will denote these coefficients simply as $E_{j 1}$. Notice that these terms include in principle $E_{j 1}^{I}$ and $E_{j 1}^{I I}$, but $E_{r 1}^{I I}=E_{r+1,1}^{I I}=0$ because the starting procedure has order at least $r+2$. Also notice that in our case the monodromy matrix in the same formula (3.8) should be $M_{t_{0}}$ as $t_{0}$ is the time corresponding to $\hat{Y}_{\left[\frac{k}{2}\right]}$ now.

We will now concentrate our attention on the components of $\left(\dot{E}_{j 1}, E_{j 1}\right)^{T}$ in $\operatorname{ker}\left(M_{t_{0}}-I\right)^{2}$, as the other components will grow linearly because of Lemma 3.3 For brevity, we will also denote as $E_{j 1}$ that component. Taking into account that [5]

$$
\operatorname{ker}\left(M_{t_{0}}-I\right)^{2}=\left(\operatorname{ker}\left(M_{t_{0}}-I\right)^{2} \cap X_{+}\right) \oplus\left(\operatorname{ker}\left(M_{t_{0}}-I\right)^{2} \cap X_{-}\right),
$$

we can consider the following decomposition

$$
\left(\begin{array}{c}
\dot{E}_{j 1}[1] \\
E_{j 1}[1]
\end{array}\right)=v_{-}+v_{+}+w_{+}
$$

with $v_{-}$and $v_{+}$eigenvectors of $M_{t_{0}}$ in $X_{-}$and $X_{+}$, respectively, and $w_{+}$a generalized eigenvector in $X_{+}$.

Then, for $j=r$, (3.8) with $N=1$ says that

$$
-\pi_{2}\left(v_{-}+v_{+}+w_{+}\right)=\pi_{2} M_{t_{0}}-\left(-v_{-}+v_{+}+w_{+}\right)=\pi_{2}\left(-v_{-}+v_{+}+w_{+}+c_{-}\right),
$$

where $c_{-}$is an eigenvector in $X_{-}$[5] calculated as $c_{-}=M_{t_{0}} w_{+}-w_{+}$. From this equality, as $\pi_{2}\left(v_{+}+w_{+}\right) \in \bar{X}_{+}$and $\pi_{2} c_{-} \in \bar{X}_{-}$, each of them vanishes. The 
consequence this has on $E_{j 1}[N]$ is the following:

$$
\begin{aligned}
\left(\begin{array}{c}
\dot{E}_{j 1}[N] \\
E_{j 1}[N]
\end{array}\right) & =\left(\sum_{l=0}^{N-1} M_{t_{0}}^{l}\right)\left(\begin{array}{c}
\dot{E}_{j 1}[1] \\
E_{j 1}[1]
\end{array}\right) \\
& =N v_{-}+N v_{+}+N w_{+}+\frac{N(N-1)}{2} c_{-} .
\end{aligned}
$$

Therefore $E_{j 1}[N]=N \pi_{2} v_{-}$, and this term grows linearly.

For $j=r+1$, notice that $\left(I_{1, r+1}, I_{2, r+1}\right)=\left(I_{1, r+1}, 0\right) \in X_{-}$because $I_{1, r+1} \in \bar{X}_{+}$. It can be proved that its component in $\operatorname{ker}\left(M_{t_{0}}-I\right)^{2}$ is also in $X_{-}$. Therefore, this component is an eigenvector of $M_{t_{0}}$. As its second component vanishes, (3.8) says the same in situation $\left(\mathrm{R}^{\prime \prime}\right)$ as for $j=r$ and the same proof as earlier leads to linear error growth.

For $j=r+2$, if we consider again decomposition (3.12) for $\left(\dot{E}_{r+2,1}^{I}[1], E_{r+2,1}^{I}[1]\right)^{T}$, (3.13) would be true for $\left(\dot{E}_{r+2,1}^{I}[N], E_{r+2,1}^{I}[N]\right)^{T}$. As for $E_{r+2,1}^{I I}$, if we have a similar decomposition to (3.12) for the initial condition,

$$
\left(\begin{array}{l}
\dot{E}_{r+2,1}^{I I}\left(t_{0}\right) \\
E_{r+2,1}^{I I}\left(t_{0}\right)
\end{array}\right)=v_{-}^{I I}+v_{+}^{I I}+w_{+}^{I I},
$$

then

$$
\left(\begin{array}{c}
\dot{E}_{r+2,1}^{I I}[N] \\
E_{r+2,1}^{I I}[N]
\end{array}\right)=M_{t_{0}}^{N}\left(\begin{array}{c}
\dot{E}_{r+2,1}^{I I}\left(t_{0}\right) \\
E_{r+2,1}^{I I}\left(t_{0}\right)
\end{array}\right)=v_{-}^{I I}+v_{+}^{I I}+w_{+}^{I I}+N c_{-}^{I I},
$$

for $c_{-}^{I I}$ an eigenvector in $X_{-}$. Inserting all this in formula (3.8) and taking into account that $M_{t_{0}}^{N}\left(I_{1, r+2}, I_{2, r+2}\right)^{T}$ will grow at most linearly, the terms in $N^{2}$ can be equated, obtaining $\pi_{2} c_{-}=0$ for $c_{-}$in (3.13). Therefore, $E_{r+2,1}^{I}[N]$ grows linearly with $N$, as well as $E_{r+2,1}[N]$.

Let us consider now the case in which $\tilde{y}_{0}$ does not belong to $\bar{X}_{+}$. Let us assume the orbit cuts $\bar{X}_{+}$at time $t^{*} \in\left[t_{0}, t_{0}+T\right]$. Again, the coefficient to study is $E_{j 1}^{I}[N]$ $(j=r, r+1, r+2)$. But,

$$
\begin{aligned}
\left(\begin{array}{c}
\dot{E}_{j 1}^{I}[N T] \\
E_{j 1}^{I}[N T]
\end{array}\right)= & \int_{t_{0}}^{t_{0}+N T} M\left(t_{0}+N T, s\right)\left(\begin{array}{c}
B_{j 1}(s) \\
0
\end{array}\right) d s \\
= & \int_{t_{0}}^{t^{*}} M\left(t_{0}+N T, s\right)\left(\begin{array}{c}
B_{j 1}(s) \\
0
\end{array}\right) d s \\
& +\int_{t^{*}}^{t^{*}+(N-1) T} M\left(t_{0}+N T, s\right)\left(\begin{array}{c}
B_{j 1}(s) \\
0
\end{array}\right) d s \\
& +\int_{t^{*}+(N-1) T}^{t_{0}+N T} M\left(t_{0}+N T, s\right)\left(\begin{array}{c}
B_{j 1}(s) \\
0
\end{array}\right) d s .
\end{aligned}
$$

Notice now that the first integral above grows linearly because of the Jordan structure of the matrix $M_{t_{0}}$, and the third one is bounded because of the periodicity of $B_{j 1}(s)$. As for the second one, it can be interpreted as $M\left(t_{0}+T, t^{*}\right) \hat{E}_{j 1}^{I}[N-1]$, where $\hat{E}_{j 1}^{I}[N-1]$ corresponds to the coefficient $E_{j 1}^{I}$ of the asymptotic expansion of the global error when integrating an orbit which starts at $Y\left(t^{*}\right)$. From above we know that this term grows linearly, and therefore the result is proved for general $\tilde{y}_{0}$. (For a more detailed discussion of the interpretation of the integrals above, see the remark after Theorem 5.1 in [5], as its proof is very similar to that one.) 
Remark 3.14. We remark that an analysis similar to the one given above does lead to quadratic error growth in general for $E_{r+3}[N]$. In the experiments done for problems under hypotheses (R2 $\left.2^{\prime \prime}\right)$, linear error growth with time is observed for a very long time, but not as long as for their FSLMM2s counterparts. This is explained by the fact that $E_{r+3}[N]$ grows linearly with $N$ for fixed stepsizes, as was proved in [7].

Remark 3.15. The terms in $E_{r}[N]$ and $E_{r+1}[N]$ which grow linearly are in $\bar{X}_{-}$. In this way, for each problem, the type of geometric errors committed can be determined.

\section{Conditions for a VSLMM2 to Be Symmetric}

Given the results in section 3, we are very much interested in the construction of symmetric VSLMM2 in order to integrate reversible systems of the form (2.6). For that purpose, the following characterization of this type of methods is extremely useful.

Theorem 4.1. The necessary and sufficient conditions for a VSLMM2 of the form (2.7) to be symmetric are the following,

$$
\begin{aligned}
& A_{0}\left(h_{n}, \ldots, h_{n+k-1}\right) A_{l}\left(h_{n+k-1}, \ldots, h_{n}\right) \\
& \quad=A_{k-l}\left(h_{n}, \ldots, h_{n+k-1}\right) A_{k}\left(h_{n+k-1}, \ldots, h_{n}\right), \quad l=0, \ldots, k-1, \\
& \quad \begin{array}{l}
A_{0}\left(h_{n}, \ldots, h_{n+k-1}\right) B_{l}\left(h_{n+k-1}, \ldots, h_{n}\right) \\
\quad=\frac{h_{n}^{2}}{h_{n+k-1}^{2}} B_{k-l}\left(h_{n}, \ldots, h_{n+k-1}\right) A_{k}\left(h_{n+k-1}, \ldots, h_{n}\right), \quad l=0, \ldots, k,
\end{array}
\end{aligned}
$$

for every choice of stepsizes $h_{n}, \ldots, h_{n+k-1}$, such that $A_{0}\left(h_{n}, \ldots, h_{n+k-1}\right) \neq 0$ and $A_{k}\left(h_{n+k-1}, \ldots, h_{n}\right) \neq 0$.

Proof. To prove that these conditions are sufficient, it suffices to notice that whenever the method takes $Y_{n}, \ldots, Y_{n+k-1}$ to $Y_{n+k}$ through (2.7), conditions (4.1) and (4.2) imposed in the same equation say that

$$
\begin{aligned}
& \frac{A_{k}\left(h_{n+k-1}, \ldots, h_{n}\right)}{A_{0}\left(h_{n}, \ldots, h_{n+k-1}\right)} \sum_{l=0}^{k} A_{k-l}\left(h_{n}, \ldots, h_{n+k-1}\right) Y_{n+l} \\
& \quad=\frac{A_{k}\left(h_{n+k-1}, \ldots, h_{n}\right)}{A_{0}\left(h_{n}, \ldots, h_{n+k-1}\right)} h_{n}^{2} \sum_{l=0}^{k} B_{k-l}\left(h_{n}, \ldots, h_{n+k-1}\right) F\left(Y_{n+l}\right) .
\end{aligned}
$$

From this, the quotient can be cancelled, which states that the method would take $Y_{n+k}, \ldots, Y_{n+1}$ to $Y_{n}$, if the same stepsizes in the reversed order had been considered, i.e., the method is symmetric.

In order to prove that these conditions are also necessary, notice that the following values

$$
Y_{n}=0, \ldots, Y_{n+l}=1, \ldots, Y_{n+k-1}=0, \quad f=0,
$$

lead to $Y_{n+k}=-A_{l}\left(h_{n+k-1}, \ldots, h_{n}\right) / A_{k}\left(h_{n+k-1}, \ldots, h_{n}\right)$. On the other hand, because of symmetry,

$$
A_{k-l}\left(h_{n}, \ldots, h_{n+k-1}\right)+A_{0}\left(h_{n}, \ldots, h_{n+k-1}\right) Y_{n+k}=0,
$$


from which (4.1) follows for $l=0, \ldots, k-1$. Besides, for the same starting values, if

$$
f=0 \text { except in } 1, \quad f(1)=1,
$$

then

$$
Y_{n+k}=\frac{h_{n+k-1}^{2} B_{l}\left(h_{n+k-1}, \ldots, h_{n}\right)-A_{l}\left(h_{n+k-1}, \ldots, h_{n}\right)}{A_{k}\left(h_{n+k-1}, \ldots, h_{n}\right)},
$$

and because of symmetry,

$$
A_{k-l}\left(h_{n}, \ldots, h_{n+k-1}\right)+A_{0}\left(h_{n}, \ldots, h_{n+k-1}\right) Y_{n+k}=h_{n}^{2} B_{k-l}\left(h_{n}, \ldots, h_{n+k-1}\right),
$$

from which (4.2) follows for $l=0, \ldots, k-1$ using (4.1). Finally, for $l=k$, it suffices to consider the same condition for $l=0$ in the reversed order and apply (4.1) for $l=0$.

Remark 4.2. Notice that, for fixed stepsizes, $A_{0}=A_{k} \neq 0$, and as we will see from (A.1), $A_{0}\left(h_{n+k-1}, \ldots, h_{n}\right)$ and $A_{k}\left(h_{n}, \ldots, h_{n+k-1}\right)$ do not vanish for $\epsilon$ small enough when the stepsizes are taken as in (1.2).

For practical purposes, we give the following sufficient conditions for symmetry, much easier to verify and impose than (4.1) and (4.2).

Corollary 4.3. A sufficient condition for a VSLMM2 to be symmetric is

$$
\begin{aligned}
A_{l}\left(h_{n+k-1}, \ldots, h_{n}\right) & =A_{k-l}\left(h_{n}, \ldots, h_{n+k-1}\right), \quad l=0, \ldots, k, \\
B_{l}\left(h_{n+k-1}, \ldots, h_{n}\right) & =\frac{h_{n}^{2}}{h_{n+k-1}^{2}} B_{k-l}\left(h_{n}, \ldots, h_{n+k-1}\right), \quad l=0, \ldots, k .
\end{aligned}
$$

Remark 4.4. Notice that, for fixed-stepsize methods, conditions (4.1), (4.2) and stability imply the well-known condition [20]

$$
A_{l}=A_{k-l}, \quad B_{l}=B_{k-l}, \quad l=0, \ldots, k,
$$

which is what (4.3) says for fixed stepsizes.

\section{Numerical EXAmPles}

In this section we corroborate some of the results proved in previous ones. We will be integrating Kepler's problem,

$$
\begin{aligned}
& \ddot{y}_{i}=-\frac{y_{i}}{\left(y_{1}^{2}+y_{2}^{2}\right)^{\frac{3}{2}}}, \\
& y_{1}(0)=1-e, \quad y_{2}(0)=0, \quad \dot{y}_{1}(0)=0, \quad \dot{y}_{2}(0)=\sqrt{\frac{1+e}{1-e}},
\end{aligned}
$$

with eccentricity $e=0.9$ in order to profit from the adaptivity of our integrators. Notice that this problem is reversible with respect to the involution

$$
\Lambda\left(\dot{y}_{1}, \dot{y}_{1}, y_{1}, y_{2}\right)^{T}=\left(-\dot{y}_{1}, \dot{y}_{1}, y_{1},-y_{2}\right)^{T}
$$

and that the orbit in which we are interested (an ellipse) is symmetric with respect to this involution, because it cuts its invariant subspace. On the other hand, as studied in [5], the Jordan structure of the monodromy matrix $M_{0}$ associated to (5.1) puts this problem under situation $\left(\mathrm{R} 2^{\prime \prime}\right)$. 


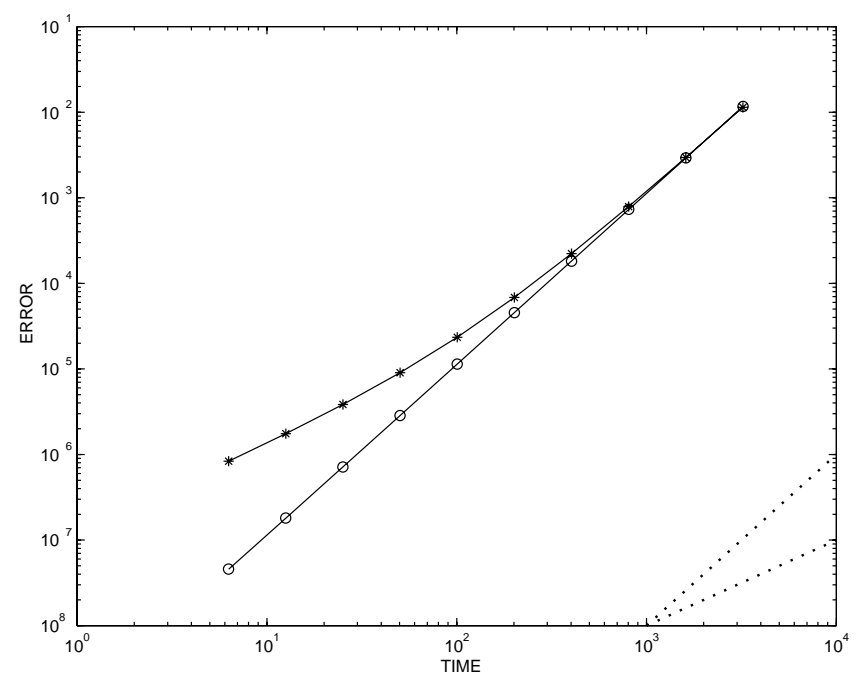

FIgURE 1. Error growth with Adams methods of orders 4 and 5.

5.1. Adams methods. First, we write (5.1) as a first-order system and integrate it with the variable-stepsize Adams methods of orders 4 and 5 [11], taking as stepsize function

$$
s\left(\dot{y}_{1}, \dot{y}_{2}, y_{1}, y_{2}\right)=\frac{\pi}{2 \sqrt{2}}\left(y_{1}^{2}+y_{2}^{2}\right)^{\frac{3}{4}} .
$$

Notice that this is small in the pericentre and large in the apocentre. (This choice was suggested in [5] and is the time of a free fall into the centre from the current configuration.) The methods have been implemented following the indications in [1]. Figure 1 shows how error grows with time when measuring the error at final times $T, 2 T, \ldots, 512 T$, with $T=2 \pi$ the period of the problem, taking as a tolerance $\epsilon=\pi 10^{-3}$. Asterisks and circles correspond to the 4th- and 5th-order methods, respectively. As expected by Remark 3.8, the error growth with time for the 4thorder integrator is linear at the beginning and then becomes quadratic, while for the 5th-order integrator is quadratic from the very beginning.

\subsection{Symmetric LMM2s.}

5.2.1. Construction. We consider 4th-step 4th-order FSLMM2s which are explicit and symmetric and then construct explicit symmetric VSLMM2's whose restriction at fixed stepsize coincide with the first ones. According to Remark 2.11 it suffices to impose order 2 over the VSLMM2 to obtain order 4 in fact. In such a case, the conditions of order would be 4 and we have eight unknowns $A_{i}\left(h_{n+3}, \ldots, h_{n}\right)(i=$ $0, \ldots, 4), B_{i}\left(h_{n+3}, \ldots, h_{n}\right)(i=1,2,3)$. As the symmetry conditions are difficult to treat by themselves because the kind of dependence of the coefficients on the stepsizes is not established, we have forced symmetry to some extent by writing the order conditions in forward and backward forms and assuming then conditions (4.3). More explicitly, we have made formula (2.7) exact for the polynomials $y(t)=t^{m}$ $(m=0,1,2,3)$, when going from $t_{0}=0$ to $t_{4}=h_{0}+h_{1}+h_{2}+h_{3}$ in this order and in the reversed one, $t_{4}=h_{3}+h_{2}+h_{1}+h_{0}$. In such a way, a linear system of seven 
different equations and eight unknowns turn up, whose solution is

$$
\begin{aligned}
A_{4}= & -A_{1} \frac{h_{0}}{h_{0}+h_{1}+h_{2}+h_{3}}-A_{2} \frac{h_{0}+h_{1}}{h_{0}+h_{1}+h_{2}+h_{3}}-A_{3} \frac{h_{0}+h_{1}+h_{2}}{h_{0}+h_{1}+h_{2}+h_{3}}, \\
A_{0}= & -A_{3} \frac{h_{3}}{h_{0}+h_{1}+h_{2}+h_{3}}-A_{2} \frac{h_{2}+h_{3}}{h_{0}+h_{1}+h_{2}+h_{3}}-A_{1} \frac{h_{1}+h_{2}+h_{3}}{h_{0}+h_{1}+h_{2}+h_{3}}, \\
B_{1}= & B_{3} \frac{h_{2}}{h_{1}}-A_{3} g_{3}\left(h_{3}, h_{2}, h_{1}, h_{0}\right)-A_{2} g_{2}\left(h_{3}, h_{2}, h_{1}, h_{0}\right)-A_{1} g_{1}\left(h_{3}, h_{2}, h_{1}, h_{0}\right), \\
B_{2}= & -B_{3} \frac{h_{1}+h_{2}}{h_{1}}-A_{3} p_{3}\left(h_{3}, h_{2}, h_{1}, h_{0}\right) \\
& -A_{2} p_{2}\left(h_{3}, h_{2}, h_{1}, h_{0}\right)-A_{1} p_{1}\left(h_{3}, h_{2}, h_{1}, h_{0}\right) .
\end{aligned}
$$

Here, the coefficients $A_{j}, B_{j}$ are evaluated at $\left(h_{3}, h_{2}, h_{1}, h_{0}\right)$ and the formulas are the same when substituted by $\left(h_{n+3}, h_{n+2}, h_{n+1}, h_{n}\right)$. On the other hand, $\left\{g_{i}\right\}$ and $\left\{p_{i}\right\}$ are rational functions of the stepsizes.

The procedure will be to substitute the fixed coefficients $A_{1}, A_{2}, A_{3}$ in the equations above and to get from there the functions $A_{0}(), A_{4}(), B_{1}(), B_{2}(), B_{3}()$. For $B_{i}$, we also assume $6 h_{0}^{2} h_{2} B_{3}($ ) has a homogeneous 3rd-degree polynomial expression on the stepsizes and impose again the symmetry conditions and their coincidence with their fixed-stepsize counterparts. Notice from (5.3) that (2.8) is satisfied. (In fact, the derivation with respect to stepsizes of the equations of consistency leads to it in general.)

We have made two different choices for the FSLMM2s. Notice that all 4thstep 4th-order symmetric explicit stable FSLMM2's correspond to characteristic polynomials

$$
\begin{aligned}
R(x) & =\left(x^{2}+a x+1\right)(x-1)^{2}, \\
S(x) & =b_{1} x^{3}+b_{2} x^{2}+b_{1} x,
\end{aligned}
$$

with $|a| \leq 2$, and

$$
\begin{aligned}
2+a & =2 b_{1}+b_{2}, \\
28+2 a & =24 b_{1} .
\end{aligned}
$$

Then, $a=2$ minimizes the local error. With this value of $a$, the resulting FSLMM2 has $R(-1)=R^{\prime}(-1)=0$, and therefore there is not an advantageous error growth for fixed stepsizes. With variable ones (and $A_{4} \neq 1$ ), -1 is just a single root of

$$
A_{4}\left(h_{3}, h_{2}, h_{1}, h_{0}\right) x^{4}+A_{3} x^{3}+A_{2} x^{2}+A_{1} x+A_{0}\left(h_{3}, h_{2}, h_{1}, h_{0}\right),
$$

and we are interested in looking at error growth also in this case. On the other hand, if $a=(19 / 10)$ (quite near 2), the corresponding FSLMM2 has just 1 double root and its error growth for this problem is linear. We are interested in corroborating that the same happens with variable stepsizes.

5.2.2. Numerical results. We have implemented both methods using stabilization [11] and compensated summation, in order to reduce roundoff error as much as possible. The stepsize function has been chosen in a symmetric way according to (3.6) taking as $\tau$ the function in (5.2). We have solved the implicitness in (3.61) by fixed point iteration, stopping it when the relative difference between two consecutive approximations is less than $10^{-3}$. As starting values we have taken the exact ones for our double precision. 


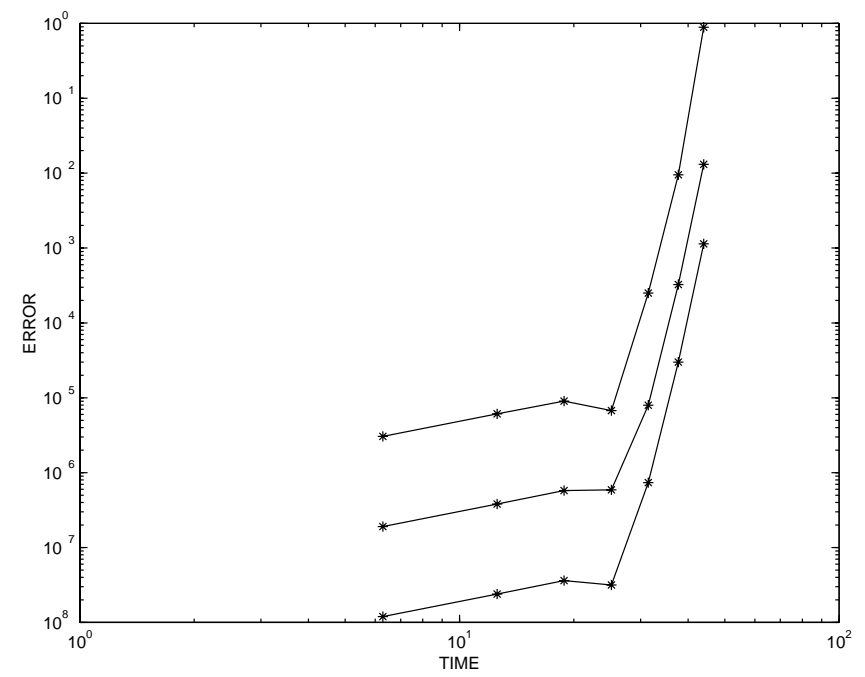

FIGURE 2. Error growth with VSLMM2 corresponding to $a=2$

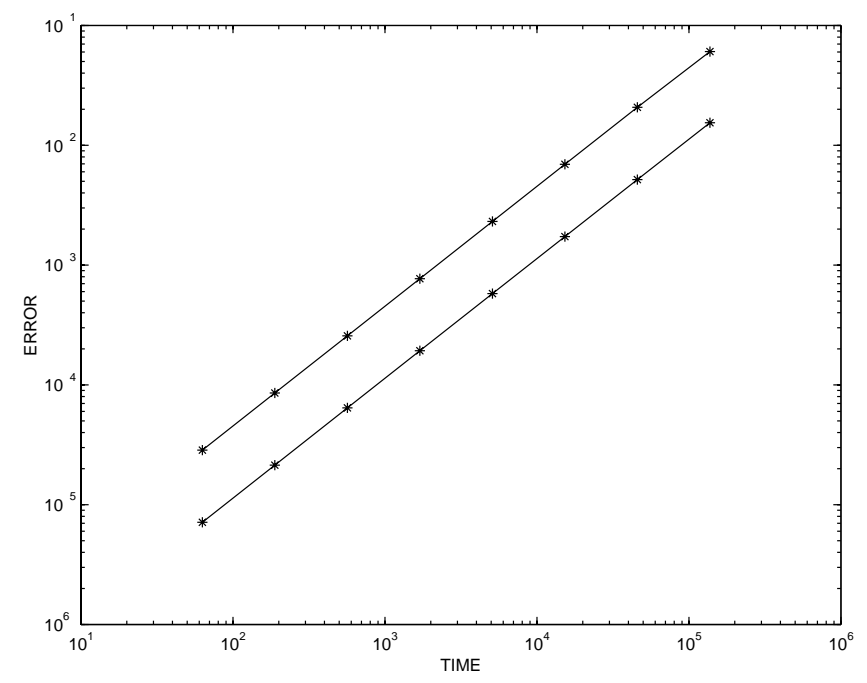

FIGURE 3. Error growth with VSLMM2 corresponding to $a=19 / 10$

For $a=2$, we have considered different values of $\epsilon\left(2 \pi /\left(2^{j} 10^{3}\right), j=0,1,2\right)$. Each line in Figure 2 corresponds to one of them. Notice that error growth is exponential, as corresponds to the analysis given in subsection 3.2.1. For the smallest value of $\epsilon$, the error increases in five orders of magnitude in just 7 periods.

However, for $a=19 / 10$ and $\epsilon=2 \pi \times 10^{-6}, \pi \times 10^{-6}$, linear error growth is observed for very long times in Figure 3. The asterisks there correspond to errors measured at times $10 T, 30 T, 90 T, \ldots, 21870 T$. Therefore, the results corroborate Theorem 3.13. As the gradient of momentum and energy are symmetric for this problem [5], we can assure in addition that the projections of errors in both quantities are bounded with respect to the terms in $\epsilon^{r}, \epsilon^{r+1}$ (see Remark 3.15). 
On the other hand, order 4 is manifest in both figures. When $\epsilon$ is halved, the error is divided approximately by 16 for a given time. This corroborates Remark 2.11.

\section{Concluding Remarks}

The use of explicit symmetric VSLMM2s with no parasitic root is very much recommended for the integration of eccentric orbits of reversible systems of the type (2.6), (3.5). The techniques in [11] 15, 17] to reduce the integration to a fixedstepsize integration through a reparameterization cannot be applied here because the new system to integrate would not have the form (2.6) any more. On the other hand, in spite of having to calculate the coefficients of the method each time the stepsize changes and solve implicitly for the stepsize, just one function evaluation per step is needed, in contrast with variable-stepsize Runge-Kutta-Nyström methods which would require new function evaluations every time a stepsize is rejected. This makes VSLMM2s very much suitable to integrate large problems where the function evaluation is very costly, and the advantage over one-step methods will be more noticeable when the order of the integration is larger. The development of

efficient techniques to construct the variable coefficients of high-order VSLMM2s is an object of further research [4].

\section{Appendix A. Technical proofs of Section 2}

A.1. Proof of Lemma 2.1. The result for constant $\left\{\alpha_{l}\right\}$ satisfying the stability condition for FSLMMs is given in [14]. To make it extensible now, it suffices to notice, taking (2.2) into account, that

(A.1)

$$
\begin{aligned}
\alpha_{l}( & \left.h_{n+k-1}, \ldots, h_{n}\right) \\
= & \alpha_{l}\left(\epsilon s\left(y\left(t_{n}\right), \epsilon\right), \ldots, \epsilon s\left(y\left(t_{n}\right), \epsilon\right)\right) \\
& +\epsilon \sum_{m=0}^{k-1} \frac{\partial \alpha_{l}}{\partial h_{m}}\left(\epsilon s\left(y\left(t_{n}\right), \epsilon\right), \ldots, \epsilon s\left(y\left(t_{n}\right), \epsilon\right)\right)\left(s\left(y\left(t_{n+m}\right), \epsilon\right)-s\left(y\left(t_{n}\right), \epsilon\right)\right)+\cdots \\
= & \alpha_{l}+\epsilon^{2} s\left(y\left(t_{n}\right), \epsilon\right) s_{y}\left(y\left(t_{n}\right), \epsilon\right) \dot{y}\left(t_{n}\right) \sum_{m=0}^{k-1} \frac{\partial \alpha_{l}}{\partial h_{m}} m+O\left(\epsilon^{2}\right) \\
= & \alpha_{l}+\epsilon s_{y}\left(y\left(t_{n}\right), \epsilon\right) f\left(y\left(t_{n}\right)\right) \sum_{m=0}^{k-1} \alpha_{l, m}^{D} m+O\left(\epsilon^{2}\right) \\
= & \alpha_{l}+O(\epsilon) .
\end{aligned}
$$

Therefore, introducing the residual $O(\epsilon)$ in the second member of (2.4), the only thing to do is to refer to the result in [14].

A.2. Proof of Theorem 2.2. For simplicity we will assume every root of $\rho$ is single and nonzero. The extension to the general case would be quite straightforward.

Let us consider the sequence $\delta_{n}$

$$
\delta_{n}=y_{n}-y\left(t_{n}\right)-\sum_{j=r}^{2 r-1} \epsilon^{j} \sum_{i=1}^{k} x_{i}^{n} e_{j i}\left(t_{n}\right),
$$


for some differentiable functions $\left\{e_{j i}\right\}$. As the method has order $r$, it is obvious that $\delta_{n}=O\left(\epsilon^{r}\right)(\epsilon \rightarrow 0)$. We will consider the following notation for (2.1):

$$
0=\rho_{n, \epsilon}(E) y_{n}-h_{n+k-1} \sigma_{n, \epsilon}(E) f\left(y_{n}\right) .
$$

Then, by substituting $y_{n}$ for the corresponding expression from A.2 , we have

$$
\begin{aligned}
0= & \rho_{n, \epsilon}(E) y\left(t_{n}\right)-h_{n+k-1} \sigma_{n, \epsilon}(E) f\left(y\left(t_{n}\right)\right) \\
& +\sum_{j=r}^{2 r-1} \epsilon^{j}\left[\rho_{n, \epsilon}(E) e_{j 1}\left(t_{n}\right)-h_{n+k-1} \sigma_{n, \epsilon}(E) f^{\prime}\left(y\left(t_{n}\right)\right) e_{j 1}\left(t_{n}\right)\right] \\
& +\sum_{i=2}^{k} x_{i}^{n} \sum_{j=r}^{2 r-1} \epsilon^{j}\left[\rho_{n, \epsilon, i}(E) e_{j i}\left(t_{n}\right)-h_{n+k-1} \sigma_{n, \epsilon, i}(E) f^{\prime}\left(y\left(t_{n}\right)\right) e_{j i}\left(t_{n}\right)\right] \\
& +\rho_{n, \epsilon}(E) \delta_{n}-h_{n+k-1} \sigma_{n, \epsilon}(E) f^{\prime}\left(y\left(t_{n}\right)\right) \delta_{n} \\
& +h_{n+k-1} O\left(\epsilon^{2 r}\right) .
\end{aligned}
$$

Here $\rho_{n, \epsilon, i}$ and $\sigma_{n, \epsilon, i}$ correspond to the operators with variable stepsizes with coefficients $\left\{\alpha_{l}\left(h_{n+k-1}, \ldots, h_{n}\right) x_{i}^{l}\right\}$ and $\left\{\beta_{l}\left(h_{n+k-1}, \ldots, h_{n}\right) x_{i}^{l}\right\}$. See that the first term here corresponds to the local truncation error of the method. By developing the terms $y\left(t_{n+l}\right)$ and $\dot{y}\left(t_{n+l}\right)$ in it around $t_{n}$, and taking into account that the formula is exact for polynomials of degree $\leq r$, this term has the form

$$
\sum_{j=r}^{\infty} y^{(j+1)}\left(t_{n}\right) d_{j}\left(h_{n+k-1}, \ldots, h_{n}\right),
$$

where

$$
\begin{aligned}
d_{j}\left(h_{n+k-1}, \ldots, h_{n}\right)= & \frac{1}{(j+1) !} \sum_{l=0}^{k} \alpha_{l}\left(h_{n+k-1}, \ldots, h_{n}\right)\left(h_{n}+\cdots+h_{n+l-1}\right)^{j+1} \\
& -\frac{h_{n+k-1}}{j !} \sum_{l=0}^{k} \beta_{l}\left(h_{n+k-1}, \ldots, h_{n}\right)\left(h_{n}+\cdots+h_{n+l-1}\right)^{j} .
\end{aligned}
$$

Therefore, the first term in (A.3) can also be written as

$$
h_{n+k-1} \sum_{j=r}^{2 r-1} \epsilon^{j} \tilde{d}_{j}\left(t_{n}\right)+O\left(\epsilon^{2 r+1}\right),
$$

for smooth functions $\left\{\tilde{d}_{j}\right\}$ which depend on the stepsize selection function $s$, the exact solution $y(\cdot)$ and the coefficients of the method. Notice also that whenever $y(\cdot)$ is periodic, these functions will also be periodic. On the other hand, it is also possible and convenient for us to write this expression as

$$
-h_{n+k-1} \sigma_{n, \epsilon}(E) \sum_{j=r}^{2 r-1} \epsilon^{j} b_{j 1}\left(t_{n}\right)+O\left(\epsilon^{2 r+1}\right),
$$

for some functions $b_{j 1}$ which satisfy the same properties as the $\tilde{d}_{j}$ before. In case $j=r$, notice that $\tilde{d}_{r}=c_{r} y^{(r+1)}$ and therefore $b_{r 1}=-c_{r} y^{(r+1)}$. 
By considering the third line in (A.3), for each index $i=2, \ldots, k$, the sum in $j$ can be written except for a $O\left(\epsilon^{2 r+1}\right)$-term as

$$
\sum_{j=r}^{2 r-1} \epsilon^{j}\left[\bar{\rho}_{n, \epsilon, i}(E) e_{j i}\left(t_{n}\right)-\epsilon s\left(y\left(t_{n}\right), \epsilon\right) \bar{\sigma}_{n, \epsilon, i}\left(F^{\prime}\left(y\left(t_{n}\right)\right) e_{j i}\left(t_{n}\right)+h_{j i}\left(t_{n}\right)\right)\right],
$$

where $\bar{\rho}_{n, \epsilon, i}$ and $\bar{\sigma}_{n, \epsilon, i}$ are like $\rho_{n, \epsilon, i}$ and $\sigma_{n, \epsilon, i}$ but associated to fixed stepsize $\epsilon s\left(y\left(t_{n}\right), \epsilon\right)$. The difference between them leads to the terms $\left\{h_{j i}\left(t_{n}\right)\right\}$. Notice that these functions will depend again on $s, y$ and their derivatives and the coefficients of the integrator considered, as well as on $e_{j i}\left(t_{n}\right)$ and $e_{l i}\left(t_{n}\right)$ and its derivatives for $r \leq l<j$.

Because of all of this, A.3 is equivalent to

$$
\begin{aligned}
0= & \sum_{j=r}^{2 r-1} \epsilon^{j}\left[\rho_{n, \epsilon}(E) e_{j 1}\left(t_{n}\right)-h_{n+k-1} \sigma_{n, \epsilon}(E) \dot{e}_{j 1}\left(t_{n}\right)\right] \\
& +\sum_{j=r}^{2 r-1} \epsilon^{j} h_{n+k-1} \sigma_{n, \epsilon}(E)\left[\dot{e}_{j 1}\left(t_{n}\right)-f^{\prime}\left(y\left(t_{n}\right)\right) e_{j 1}\left(t_{n}\right)-b_{j 1}\left(t_{n}\right)\right] \\
& +\sum_{i=2}^{k} x_{i}^{n} \sum_{j=r}^{2 r-1} \epsilon^{j}\left[\bar{\rho}_{n, \epsilon, i}(E) e_{j i}\left(t_{n}\right)-\epsilon s\left(y\left(t_{n}\right), \epsilon\right) \tilde{\bar{\sigma}}_{n, \epsilon, i} \dot{e}_{j i}\left(t_{n}\right)\right] \\
& +\sum_{i=2}^{k} x_{i}^{n} \sum_{j=r}^{2 r-1} \epsilon^{j+1} s\left(y\left(t_{n}\right), \epsilon\right) \tilde{\bar{\sigma}}_{n, \epsilon, i} \\
& \quad \times\left[\dot{e}_{j i}\left(t_{n}\right)-\lambda_{i}\left(f^{\prime}\left(y\left(t_{n}\right)\right) e_{j i}\left(t_{n}\right)+h_{j i}\left(t_{n}\right)\right)\right] \\
& +\rho_{n, \epsilon}(E) \delta_{n}-h_{n+k-1} \sigma_{n, \epsilon}(E) f^{\prime}\left(y\left(t_{n}\right)\right) \delta_{n} \\
& +O\left(\epsilon^{2 r+1}\right) .
\end{aligned}
$$

Here $\tilde{\bar{\sigma}}_{n, \epsilon, i}=\bar{\sigma}_{n, \epsilon, i} / \lambda_{i}$, in such a way that the method $\left(\bar{\rho}_{n, \epsilon, i}, \tilde{\bar{\sigma}}_{n, \epsilon, i}\right)$ is consistent because

$$
\bar{\rho}_{n, \epsilon, i}^{\prime}(1)=x_{i} \rho^{\prime}\left(x_{i}\right)=\bar{\sigma}_{n, \epsilon, i}(1) \frac{x_{i} \rho^{\prime}\left(x_{i}\right)}{\sigma\left(x_{i}\right)}=\tilde{\bar{\sigma}}_{n, \epsilon, i}(1) .
$$

Now, the first line in (A.4) is $O\left(\epsilon^{2 r+1}\right)$ because of the order $r$ of the method. On the other hand, due to consistency, each bracket in the third line can be written as

$$
\epsilon s\left(y\left(t_{n}\right), \epsilon\right) \sum_{p=1}^{r-1} \epsilon^{p} \tilde{\bar{\sigma}}_{n, \epsilon, i}(E) g_{p, j, i}\left(t_{n}\right)+O\left(\epsilon^{r+1}\right),
$$

for functions $g_{p, j, i}\left(t_{n}\right)$ which depend on $s, y$ and $e_{j i}$ and their derivatives. Inserting these terms in the fourth line of (A.4), the latter becomes

$$
\sum_{i=2}^{k} x_{i}^{n} \sum_{j=r}^{2 r-1} \epsilon^{j+1} s\left(y\left(t_{n}\right), \epsilon\right) \tilde{\bar{\sigma}}_{n, \epsilon, i}(E)\left[\dot{e}_{j i}\left(t_{n}\right)-\lambda_{i}\left(f^{\prime}\left(y\left(t_{n}\right)\right) e_{j i}\left(t_{n}\right)+h_{j i}\left(t_{n}\right)\right)-b_{j i}\left(t_{n}\right)\right],
$$

for functions $b_{j i}$ depending on $s, y, e_{l i}(r \leq l<j)$ and their derivatives. Therefore, if (2.5) is satisfied, (A.4) is equivalent to

$$
\rho_{n, \epsilon}(E) \delta_{n}-h_{n+k-1} \sigma_{n, \epsilon}(E) f^{\prime}\left(y\left(t_{n}\right)\right) \delta_{n}=O\left(\epsilon^{2 r+1}\right) .
$$

Now, if $\delta_{\nu}=O\left(\epsilon^{2 r}\right)$, by applying Lemma $2.1 \delta_{n}=O\left(\epsilon^{2 r}\right)$ and the theorem will be proved just by taking into account that the powers $x_{i}^{n}(i=m+1, \ldots, k)$ go to zero more quickly than any power of $\epsilon$ when $\epsilon \rightarrow 0, t_{n}$ fixed. It is possible to get $\delta_{\nu}$ as small as that just by considering appropiate values for $e_{j i}\left(t_{0}\right)(i=1, \ldots, k)$. 
A.3. Proof of Lemma 2.5. Let us first prove the following.

Lemma A.1. The sequence $\left\{Z_{m}\right\}$ obtained from (2.9) satisfies

$$
Z_{n+l}=Z_{n}+O(\epsilon) Z_{n}+O\left(\epsilon^{r}\right), \quad l=0,1, \ldots, k, \quad n \geq 0,
$$

where the implicit constants in $O(\epsilon)$ and $O\left(\epsilon^{r}\right)$ are uniformly bounded in $n$.

Proof. First note that once it is proved for a particular $n$ and $l=0,1, \ldots, k-1$, it is also proved for that $n$ and $l=k$. Notice that a similar formula to (A.1) is also valid for $A_{l}$. From this and formula (2.7), we have

$$
\begin{aligned}
& {\left[A_{k}\left(h_{n+k-1}, \ldots, h_{n}\right)-h_{n+k-1}^{2} B_{k, n}\right] Z_{n+k}} \\
& =-\sum_{l=0}^{k-1} A_{l}\left(h_{n+k-1}, \ldots, h_{n}\right) Z_{n+l}+h_{n+k-1}^{2} \sum_{l=0}^{k-1} B_{l, n} Z_{n+l}+O\left(\epsilon^{r+2}\right) \\
& =-\sum_{l=0}^{k-1}\left(A_{l}+O(\epsilon)\right)\left(Z_{n}+O(\epsilon) Z_{n}+O\left(\epsilon^{r}\right)\right) \\
& \quad+h_{n+k-1}^{2} \sum_{l=0}^{k-1} B_{l, n}\left(Z_{n}+O(\epsilon) Z_{n}+O\left(\epsilon^{r}\right)\right)+O\left(\epsilon^{r+2}\right) \\
& =A_{k} Z_{n}+O(\epsilon) Z_{n}+O\left(\epsilon^{r}\right),
\end{aligned}
$$

which implies

$$
Z_{n+k}=Z_{n}+O(\epsilon) Z_{n}+O\left(\epsilon^{r}\right) \quad \text { or } \quad Z_{n}=Z_{n+k}+O(\epsilon) Z_{n+k}+O\left(\epsilon^{r}\right) .
$$

Then, the result for $n=0$ comes from the bound (2.10) for the starting values. Inductively on $n$, for $l=0,1, \ldots, k-1$,

$$
\begin{aligned}
Z_{n+l}-Z_{n} & =\left(Z_{n+l}-Z_{n+l-k}\right)+\left(Z_{n+l-k}-Z_{n}\right) \\
& =\left(Z_{n+l-k}+O(\epsilon) Z_{n+l-k}\right)-\left(Z_{n+l-k}+O(\epsilon) Z_{n+l-k}\right)+O\left(\epsilon^{r}\right) \\
& =O(\epsilon) Z_{n+l-k}+O\left(\epsilon^{r}\right)=O(\epsilon) Z_{n+l}+O\left(\epsilon^{r}\right) .
\end{aligned}
$$

Lemma A.2. The sequence $\left\{Z_{n}\right\}$ is bounded.

Proof. Notice that

$$
\sum_{l=0}^{k} \sum_{m=0}^{k-1} \frac{\partial A_{l}}{\partial h_{m}} m=\sum_{m=0}^{k-1} m \sum_{l=0}^{k} \frac{\partial A_{l}}{\partial h_{m}}=0 .
$$

The last equality comes from derivating with respect to $h_{m}$ the first condition for consistency of the variable-stepsize method. By (2.8) and (A.1), this is equivalent to

$$
\sum_{l=0}^{k} \sum_{m=0}^{k-1} A_{l, m}^{D} m=0 \quad \text { or } \quad \sum_{l=0}^{k}\left(A_{l}\left(h_{n+k-1}, \ldots, h_{n}\right)-A_{l}\right)=O\left(\epsilon^{2}\right)
$$


Now, if $S_{n, \epsilon}$ is the operator associated to the bracket in (2.9), it follows that

$$
\begin{aligned}
\sum_{l=0}^{k} A_{l}( & \left.h_{n+k-1}, \ldots, h_{n}\right) Z_{n+l}-h_{n+k-1}^{2} S_{n, \epsilon}(E) Z_{n} \\
= & R(E) Z_{n}+\sum_{l=0}^{k}\left(A_{l}\left(h_{n+k-1}, \ldots, h_{n}\right)-A_{l}\right)\left(Z_{n}+O(\epsilon) Z_{n}+O\left(\epsilon^{r}\right)\right) \\
& -h_{n+k-1}^{2} S_{n, \epsilon}(E) Z_{n} \\
= & R(E) Z_{n}+Z_{n} O\left(\epsilon^{2}\right)+Z_{n} \sum_{l=0}^{k} O(\epsilon) O(\epsilon)-h_{n+k-1}^{2} S_{n, \epsilon}(E) Z_{n}+O\left(\epsilon^{r+1}\right) \\
= & R(E) Z_{n}+Z_{n} O\left(\epsilon^{2}\right)-h_{n+k-1}^{2} S_{n, \epsilon}(E) Z_{n}+O\left(\epsilon^{r+1}\right) .
\end{aligned}
$$

Therefore, the second term can be grouped with $h_{n+k-1}^{2} S_{n, \epsilon}$ and the last one with $\lambda_{n}$, which implies that (2.9) can be written as

$$
R(E) Z_{n}-\epsilon^{2} \tilde{S}_{n, \epsilon}(E) Z_{n}=\tilde{\lambda}_{n}
$$

for an operator $\tilde{S}_{n, \epsilon}$ with coefficients $\tilde{B}_{l, n}$ again uniformly bounded and $\tilde{\lambda}_{n}=$ $O\left(\epsilon^{r+1}\right)$.

Then, defining

$$
W_{n}=\frac{1}{\epsilon} R_{2}(E) Z_{n}
$$

it satisfies

$$
R_{1}(E) W_{n}=\frac{1}{\epsilon} R(E) Z_{n}=\epsilon \tilde{S}_{n, \epsilon}(E) Z_{n}+\frac{\tilde{\lambda}_{n}}{\epsilon} .
$$

Now, if $U_{n}=\left[W_{n+m-1}^{T}, \ldots, W_{n}^{T}, Z_{n+k-m-1}^{T}, \ldots, Z_{n}^{T}\right]^{T}$,

$$
U_{n+1}=\hat{S} U_{n}+\Psi_{n}\left(U_{n}\right), \quad \text { with } \hat{S}=\left(\begin{array}{cc}
G & 0 \\
0 & H
\end{array}\right),
$$

where $G$ and $H$ are the companion matrices of $R_{1}$ and $R_{2}$, respectively, and

$$
\Psi_{n}\left(U_{n}\right)=\left[\epsilon \tilde{S}_{n, \epsilon}(E) Z_{n}^{T}+\frac{\tilde{\lambda}_{n}^{T}}{\epsilon}, 0, \ldots, 0, \epsilon W_{n}^{T}, 0, \ldots, 0\right]^{T} .
$$

Notice that $\Psi_{n}$ is in fact a function of $U_{n}$, by considering (A.6) and the fact that

$$
Z_{n+k}=\frac{1}{A_{k}-\epsilon^{2} \tilde{B}_{k, n}}\left[\tilde{\lambda}_{n}+\epsilon^{2} \sum_{l=0}^{k-1} \tilde{B}_{l, n} Z_{n+l}-\sum_{l=0}^{k-1} A_{l} Z_{n+l}\right],
$$

because of (A.5). Therefore, $\Psi_{n}\left(U_{n}\right)$ can also be written as

$$
\Psi_{n}\left(U_{n}\right)=\epsilon B_{n}(\epsilon) U_{n}+\Lambda_{n}(\epsilon)
$$

with $B_{n}(\epsilon)$ a uniformly bounded matrix and

$$
\Lambda_{n}(\epsilon)=\left[\left(\frac{\epsilon}{A_{k}-\epsilon^{2} \tilde{B}_{k, n}} \tilde{B}_{k, n}+\frac{1}{\epsilon}\right) \tilde{\lambda}_{n}^{T}(\epsilon), 0, \ldots, 0\right]^{T} .
$$

Considering (A.7) and (A.8),

$$
U_{n+1}=\hat{S} U_{n}+\epsilon B_{n}(\epsilon) U_{n}+\Lambda_{n}(\epsilon)
$$


from which it can be proved inductively that

$$
U_{n+1}=\hat{S}^{n+1} U_{0}+\epsilon \sum_{i=0}^{n}\left[\hat{S}^{n-i} B_{i}(\epsilon) U_{i}+\frac{\Lambda_{i}(\epsilon)}{\epsilon}\right] .
$$

Now, $\left\|U_{0}\right\|=O\left(\epsilon^{r}\right)$ because of (2.10), $\left\|\Lambda_{i}(\epsilon) / \epsilon\right\|=O\left(\epsilon^{r-1}\right)$ and the powers of $\hat{S}$ are bounded because of the stability of the fixed-stepsize method. Therefore, applying a Gronwall argument, $\left\|U_{n}\right\|=O\left(\epsilon^{r-1}\right)$, which implies that $\left\|Z_{n}\right\|=O\left(\epsilon^{r-1}\right)$, and $Z_{n}$ is bounded for every $n$ independently of the value of $r(\geq 1)$.

This proves stability of VSLMM2 based on stable fixed-stepsize counterparts. At the same time, see that the previous analysis could be done substituting $R$ by $R_{n, \epsilon}$, $\tilde{S}_{n, \epsilon}$ by $S_{n, \epsilon}$ and $\tilde{\lambda}_{n}$ by $\lambda_{n}$, which would be the straightforward analysis from the original scheme. In that way, we would have arrived at (A.9) with $\hat{S}$ substituted by $\hat{S}_{n, \epsilon}$ and $\left\|\Lambda_{i}(\epsilon) / \epsilon\right\|=O\left(\epsilon^{r}\right)$. The fact that $U_{n}$ is bounded implies that the multiplications $\hat{S}_{n+m, \epsilon} \ldots \hat{S}_{m, \epsilon}$ are also uniformly bounded, and applying the same argument as before, we arrive at the conclusion that $\left\|Z_{n}\right\|=O\left(\epsilon^{r}\right)$ in fact.

A.4. Proof of Theorem 2.6. For simplicity, we assume now that the roots of modulus $<1$ of $R$ are single and nonzero. We call

$$
\delta_{n}=Y_{n}-Y\left(t_{n}\right)-\sum_{j=r}^{2 r-1} \epsilon^{j}\left(\sum_{i=1}^{k-m} x_{i}^{n} E_{j i}\left(t_{n}\right)\right),
$$

and therefore the relationship (2.7) can be written as

$$
\begin{aligned}
0= & R_{n, \epsilon}(E) Y\left(t_{n}\right)-h_{n+k-1}^{2} S_{n, \epsilon}(E) F\left(Y\left(t_{n}\right)\right) \\
& +\sum_{i=1}^{k-m} x_{i}^{n} \sum_{j=r}^{2 r-1} \epsilon^{j}\left[R_{n, \epsilon, i}(E) E_{j i}\left(t_{n}\right)-h_{n+k-1}^{2} S_{n, \epsilon, i}(E) F^{\prime}\left(Y\left(t_{n}\right)\right) E_{j i}\left(t_{n}\right)\right] \\
& +R_{n, \epsilon}(E) \delta_{n}-h_{n+k-1}^{2} S_{n, \epsilon}(E) F^{\prime}\left(Y\left(t_{n}\right)\right) \delta_{n} \\
& +h_{n+k-1}^{2} O\left(\epsilon^{2 r}\right),
\end{aligned}
$$

where $R_{n, \epsilon, i}$ and $S_{n, \epsilon, i}$ are the operators associated to $R_{n, \epsilon}$ and $S_{n, \epsilon}$ with their coefficients multiplied by $x_{i}^{l}(l=0, \ldots, k)$.

See that now the local truncation error of the method can be expressed as

$$
R_{n, \epsilon}(E) Y(t)-h_{n+k-1}^{2} S_{n, \epsilon}(E) \ddot{Y}(t)=-h_{n+k-1}^{2} S_{n, \epsilon}(E)\left(\sum_{j=r}^{2 r-1} \epsilon^{j} B_{j 1}\left(t_{n}\right)\right)+O\left(\epsilon^{2 r+2}\right),
$$

for functions $B_{j 1}$ with the properties stated in the theorem. Notice also that $B_{r 1}=-C_{r} Y^{(r+2)}$.

For $i=2, \ldots, m$, the terms in the second line in A.10 can be written as

$$
\sum_{j=r}^{2 r-1} \epsilon^{j}\left[\bar{R}_{n, \epsilon, i}(E) E_{j i}\left(t_{n}\right)-\epsilon^{2} s\left(Y\left(t_{n}\right), \epsilon\right)^{2} \bar{S}_{n, \epsilon, i}(E)\left(F^{\prime}(Y(t)) E_{j i}\left(t_{n}\right)+H_{j i}\left(t_{n}\right)\right),\right.
$$

except for a $O\left(\epsilon^{2 r+2}\right)$-term, where $\bar{R}_{n, \epsilon, i}$ and $\bar{S}_{n, \epsilon, i}$ are like $R_{n, \epsilon, i}$ and $S_{n, \epsilon, i}$, but are associated to fixed stepsize $\epsilon s\left(Y\left(t_{n}\right), \epsilon\right)$. The difference between them leads to 
the functions $H_{j i}(t)$. See that $\left(\bar{R}_{n, \epsilon, i}(E)-R_{n, \epsilon, i}(E)\right) E_{j i}\left(t_{n}\right)=O\left(\epsilon^{2}\right)$ because

$$
\begin{aligned}
& \left(\bar{R}_{n, \epsilon, i}(E)-R_{n, \epsilon, i}(E)\right) E_{j i}\left(t_{n}\right) \\
& =\sum_{l=0}^{k} x_{i}^{l}\left[A_{l}-A_{l}\left(h_{n+k-1}, \ldots, h_{n}\right)\right] E_{j i}\left(t_{n}+l \epsilon s\left(Y\left(t_{n}\right), \epsilon\right)\right)+O\left(\epsilon^{2}\right) \\
& =E_{j i}\left(t_{n}\right) \sum_{l=0}^{k} x_{i}^{l}\left[A_{l}\left(h_{n+k-1}, \ldots, h_{n}\right)-A_{l}\right] \\
& \quad+\epsilon \dot{E}_{j i}\left(t_{n}\right) s\left(Y\left(t_{n}\right), \epsilon\right) \sum_{l=0}^{k} x_{i}^{l}\left[A_{l}-A_{l}\left(h_{n+k-1}, \ldots, h_{n}\right)\right] l+O\left(\epsilon^{2}\right) .
\end{aligned}
$$

The second line here is $O\left(\epsilon^{2}\right)$ because of (A.1). For the first, take $x_{i}\left(h_{n+k-1}, \ldots, h_{n}\right)$ a perturbation of $x_{i}$ such that

$$
\sum_{l=0}^{k} A_{l}\left(h_{n+k-1}, \ldots, h_{n}\right) x_{i}\left(h_{n+k-1}, \ldots, h_{n}\right)^{l}=0 .
$$

Differentiating with respect to $h_{m}$ and then evaluating at constant stepsizes,

$$
\sum_{l=0}^{k} \frac{\partial A_{l}}{\partial h_{m}} x_{i}^{l}=-\frac{\partial x_{i}}{\partial h_{m}} R^{\prime}\left(x_{i}\right)=0 .
$$

Therefore, from a formula similar to (A.1) again

$$
\sum_{l=0}^{k} x_{i}^{l}\left[A_{l}\left(h_{n+k-1}, \ldots, h_{n}\right)-A_{l}\right]=O\left(\epsilon^{2}\right) .
$$

Summing up, A.10 is equivalent to

$$
\begin{aligned}
0= & \sum_{j=r}^{2 r-1} \epsilon^{j}\left[R_{n, \epsilon}(E) E_{j 1}\left(t_{n}\right)-h_{n+k-1}^{2} S_{n, \epsilon}(E) \ddot{E}_{j 1}\left(t_{n}\right)\right] \\
& +h_{n+k-1}^{2} \sum_{j=r}^{2 r-1} \epsilon^{j} S_{n, \epsilon}(E)\left[\ddot{E}_{j 1}\left(t_{n}\right)-F^{\prime}\left(Y\left(t_{n}\right)\right) E_{j 1}\left(t_{n}\right)-B_{j 1}\left(t_{n}\right)\right] \\
& +\sum_{i=2}^{m} x_{i}^{n} \sum_{j=r}^{2 r-1} \epsilon^{j}\left[\bar{R}_{n, \epsilon, i}(E) E_{j i}\left(t_{n}\right)-\epsilon^{2} s\left(Y\left(t_{n}\right), \epsilon\right)^{2} \tilde{\bar{S}}_{n, \epsilon, i}(E) \ddot{E}_{j i}\left(t_{n}\right)\right] \\
(\mathrm{A} .11)+ & \sum_{i=2}^{m} x_{i}^{n} \sum_{j=r}^{2 r-1} \epsilon^{j+2} s\left(Y\left(t_{n}\right), \epsilon\right)^{2} \tilde{\bar{S}}_{n, \epsilon, i}(E)\left[\ddot{E}_{j i}\left(t_{n}\right)\right. \\
& +\sum_{i=m+1}^{k-m} x_{i}^{n} \sum_{j=r}^{2 r-1} \epsilon^{j}\left[R_{n, \epsilon, i}(E) E_{j i}\left(t_{n}\right) \quad-\epsilon^{2} s\left(Y\left(t_{n+k-1}\right), \epsilon\right)^{2} S_{n, \epsilon, i}(E) F^{\prime}\left(Y\left(t_{n}\right)\right) E_{j i}\left(t_{n}\right)\right] \\
& +R_{n, \epsilon}(E) \delta_{n}-h_{n+k-1} S_{n, \epsilon}(E) F^{\prime}\left(Y\left(t_{n}\right)\right) \delta_{n}+O\left(\epsilon^{2 r+2}\right) .
\end{aligned}
$$

Here $\tilde{\bar{S}}_{n, \epsilon, i}=\bar{S}_{n, \epsilon, i} / \mu_{i}$, in such a way that the method $\left(\bar{R}_{n, \epsilon, i}, \tilde{\bar{S}}_{n, \epsilon, i}\right)$ is consistent because

$$
\bar{R}_{n, \epsilon, i}^{\prime \prime}(1)=x_{i}^{2} R^{\prime \prime}\left(x_{i}\right)=\bar{S}_{n, \epsilon, i}(1) \frac{x_{i}^{2} R^{\prime \prime}\left(x_{i}\right)}{S\left(x_{i}\right)}=2 \tilde{\bar{S}}_{n, \epsilon, i}(1) .
$$


Therefore, due to consistency, the terms in the third line of (A.11) can be written as

$$
\epsilon^{2} s\left(Y\left(t_{n}\right), \epsilon\right)^{2} \sum_{p=1}^{r-1} \epsilon^{p} \tilde{\bar{S}}_{n, \epsilon, i}(E) G_{p, j, i}\left(t_{n}\right)+O\left(\epsilon^{r+2}\right),
$$

for suitable functions $G_{p, j, i}\left(t_{n}\right)$. Inserting these terms in the fourth line of A.11, we have for each double root $x_{i}$

$$
\sum_{j=r}^{2 r-1} \epsilon^{j+2} s\left(Y\left(t_{n}\right), \epsilon\right)^{2} \tilde{\bar{S}}_{n, \epsilon, i}(E)\left[\ddot{E}_{j i}\left(t_{n}\right)-\mu_{i}\left(F^{\prime}\left(Y\left(t_{n}\right)\right) E_{j i}\left(t_{n}\right)+H_{j i}\left(t_{n}\right)\right)-B_{j i}\left(t_{n}\right)\right],
$$

for functions $B_{j i}\left(t_{n}\right)$ as stated in the theorem.

For the terms in (A.11) associated to single roots, the first thing to notice is that

$$
\begin{aligned}
R_{n, \epsilon, i}(E) E_{j i}\left(t_{n}\right) \\
=\sum_{l=0}^{k} x_{i}^{l}\left(A_{l}+\epsilon^{2} s\left(Y\left(t_{n}\right), \epsilon\right) s_{y}\left(Y\left(t_{n}\right), \epsilon\right) \dot{Y}\left(t_{n}\right) \sum_{m=0}^{k-1} \frac{\partial A_{l}}{\partial h_{m}} m+O\left(\epsilon^{2}\right)\right) \\
\quad \times\left(E_{j i}\left(t_{n}\right)+l \epsilon s\left(Y\left(t_{n}\right), \epsilon\right) \dot{E}_{j i}\left(t_{n}\right)+O\left(\epsilon^{2}\right)\right) \\
=\epsilon s\left(Y\left(t_{n}\right), \epsilon\right) \dot{E}_{j i}\left(t_{n}\right) x_{i} R^{\prime}\left(x_{i}\right) \\
\quad+\left.\epsilon \frac{d}{d t} s(Y(t), 0)\right|_{t=t_{n}} E_{j i}\left(t_{n}\right)\left(\sum_{l=0}^{k} x_{i}^{l} \sum_{m=0}^{k-1} A_{l, m}^{D} m\right)+O\left(\epsilon^{2}\right) .
\end{aligned}
$$

Considering even more terms in the Taylor expansion, we see that

$$
\begin{aligned}
R_{n, \epsilon, i}( & E) E_{j i}\left(t_{n}\right) \\
= & \epsilon\left[\alpha_{0, i}^{1}\left(t_{n}\right) E_{j i}\left(t_{n}\right)+\alpha_{1, i}^{1}\left(t_{n}\right) \dot{E}_{j i}\left(t_{n}\right)\right] \\
& +\epsilon^{2}\left[\alpha_{0, i}^{2}\left(t_{n}\right) E_{j i}\left(t_{n}\right)+\alpha_{1, i}^{2}\left(t_{n}\right) \dot{E}_{j i}\left(t_{n}\right)+\alpha_{2, i}^{2}\left(t_{n}\right) \ddot{E}_{j i}\left(t_{n}\right)\right] \\
& +\epsilon^{3}\left[\alpha_{0, i}^{3}\left(t_{n}\right) E_{j i}\left(t_{n}\right)+\alpha_{1, i}^{3}\left(t_{n}\right) \dot{E}_{j i}\left(t_{n}\right)+\alpha_{2, i}^{3}\left(t_{n}\right) \ddot{E}_{j i}\left(t_{n}\right)+\alpha_{3, i}^{3}\left(t_{n}\right) \dddot{E}_{j i}\left(t_{n}\right)\right] \\
& +\ldots
\end{aligned}
$$

for $\alpha_{l, i}^{j}\left(t_{n}\right)$ as stated in the theorem. On the other hand, for every regular function $U$,

$$
\begin{aligned}
& s\left(Y\left(t_{n+k-1}\right), \epsilon\right)^{2} S_{n, \epsilon, i}(E) U\left(t_{n}\right) \\
&= \beta_{0, i}^{0}\left(t_{n}\right) U\left(t_{n}\right) \\
&+\epsilon\left[\beta_{0, i}^{1}\left(t_{n}\right) U\left(t_{n}\right)+\beta_{1, i}^{1}\left(t_{n}\right) \dot{U}\left(t_{n}\right)\right] \\
&+\epsilon^{2}\left[\beta_{0, i}^{2}\left(t_{n}\right) U\left(t_{n}\right)+\beta_{1, i}^{2}\left(t_{n}\right) \dot{U}\left(t_{n}\right)+\beta_{2, i}^{2}\left(t_{n}\right) \ddot{U}\left(t_{n}\right)\right] \\
&+\ldots
\end{aligned}
$$

for functions $\beta_{l, i}^{j}(t)$ again as stated.

In such a way, the coefficients of $x_{i}^{n}$ in the corresponding term of A.11) will be a sum in powers of $\epsilon$, where the coefficients of $\epsilon^{j}(r+1 \leq j \leq 2 r+1)$ will vanish when (2.14) is satisfied. If (2.13) is also true, we get that

$$
R_{n, \epsilon}(E) \delta_{n}-h_{n+k-1}^{2} S_{n, \epsilon}(E) F^{\prime}\left(Y\left(t_{n}\right)\right) \delta_{n}=O\left(\epsilon^{2 r+2}\right) .
$$

Now if $\delta_{\nu}=O\left(\epsilon^{2 r}\right)$ and $R_{2}(E) \delta_{\nu}=O\left(\epsilon^{2 r+1}\right), \delta_{n}$ is $O\left(\epsilon^{2 r}\right)$ because of Lemma[2.5, To achieve that, it suffices to consider suitable initial conditions for the differentiable functions $E_{j i}$, i.e., they must satisfy (2.16), (2.17), (2.18). 
A.5. Proof of Theorem 2.10. In the proof of Theorem 2.6 it has been shown that the term which would multiply $\epsilon^{r+1}$ in the asymptotic expansion of the local truncation error corresponding to variable stepsizes would be $C_{r-1} Y^{(r+1)}$, where $C_{r-1}$ is the scalar associated to that term in its fixed-stepsize counterpart. Therefore, $C_{r-1}=0$ because the latter has order $r$ and, as a consequence, the former also has the same order.

\section{REFERENCES}

[1] Calvo, M. P. and Hairer, E., Accurate long-term integration of dynamical systems, Appl. Num. Math., 18 (1995), pp. 95-105. MR 96f:65076

[2] Calvo, M. P., López-Marcos M. A. and Sanz-Serna, J. M., Variable step implementation of geometric integrators, Appl. Num. Math., 28 (1998), pp. 1-16. MR 99d:65236

[3] Cano, B. and García-Archilla, B., A generalization to variable stepsizes of Störmer methods for second-order differential equations, Appl. Num. Math., 19 (1996), pp. 401-417. MR 96j:65062

[4] Cano, B. and Durán, A., A technique to construct symmetric variable-stepsize lines multistep methods for second-order systems, Math. Comp., posted on May 29, 2003, PII S 00255718(03)01546-1 (to appear in print).

[5] Cano, B. and Sanz-Serna, J.M., Error growth in the numerical integration of periodic orbits, with application to Hamiltonian and reversible systems, SIAM J. Num. Anal., 34 (1997), pp. 1391-1417. MR 98i:65052

[6] Cano, B., Integración numérica de órbitas periódicas con métodos multipaso, PhD Thesis, Universidad de Valladolid, 1996.

[7] Cano, B. and Sanz-Serna, J.M., Error growth in the numerical integration of periodic orbits by multistep methods, with application to reversible systems, IMA J. Num. Anal., 18 (1998), pp. 57-75. MR 99d:65237

[8] Crouzeix, M. and Lisbona, F. J., The convergence of variable-stepsize, variable formula, multistep methods., SIAM J. Num. Anal., 21 (1984), pp. 512-534. MR 85m:65063

[9] Evans, N. W. and Tremaine, S., Linear multistep methods for integrating reversible differential equations, Astron. J 1181888 (1999).

[10] Gear, C. W. and Tu, K. W., The effect of variable mesh size on the stability of multistep methods, SIAM J. Num. Anal., 11 (1974), pp. 1025-1043. MR 51:4677

[11] Hairer, E., Nörsett, S. P. and Wanner, W. G., Solving Ordinary Differential Equations I. Nonstiff Problems, 2nd ed., Berlin, Springer, 1993. MR 94c:65005

[12] Hairer, E., Variable time step integration with symplectic methods, Appl. Numer. Math., 25 (1997), pp. 219-227. MR 99a:65083

[13] Hairer, E. and Stoffer, D., Reversible long-term integration with variable stepsizes, SIAM J. Sci. Comput., 18 (1997), pp. 257-269. MR 97m:65118

[14] Henrici, P., Discrete variable methods in ordinary differential equations, John-Wiley \& Sons, New-York-London-Sydney, 1962. MR 24:B1772

[15] Huang, W. and Leimkuhler, B. The Adaptive Verlet method, SIAM J. Sci. Comput. 18 (1997), pp. 239-256. MR 98g:65063

[16] Hut, P., Makino, J. and McMillan, S., Building a better leapfrog, Astrophys. J., 443 (1995), pp. L93-L96.

[17] Reich, S., Backward error analysis of numerical integrators, SIAM J. Numer. Anal. 36 (1999), pp. 1549-1570. MR 2000f:65060

[18] Stetter, H. J., Analysis of discretization methods for ordinary differential equations, Springer, Berlin-Heidelberg-New York, 1973. MR 54:14381

[19] Stoffer, D., Variable steps for reversible integration methods, Computing, 55 (1995), pp. 1-22. MR 96d:65132

[20] Stoffer, D., On reversible and canonical integration methods. Res. Rep. 88-05, Applied Mathematics, Eidgenössische Technische Hochschule (ETH), Zürich, 1988. 
Departamento de Matemática Aplicada y Computación, Facultad de Ciencias, UniVERSidAd de VAlladolid, Spain

E-mail address: bego@mac.mac.uva.es

Departamento de Matemática Aplicada y Computación, Facultad de Ciencias, UniVERsidad de VALladolid, SPAin

E-mail address: angel@mac.mac.uva.es 